\title{
Modeling of Spontaneous Activity in Developing Spinal Cord Using Activity-Dependent Depression in an Excitatory Network
}

\author{
Joël Tabak, ${ }^{1}$ Walter Senn, ${ }^{2}$ Michael J. O’Donovan, ${ }^{1}$ and John Rinzel ${ }^{3}$ \\ ${ }^{1}$ Laboratory of Neural Control, National Institute of Neurological Diseases and Stroke/National Institutes of Health, \\ Bethesda, Maryland 20892, ${ }^{2} P h y s i o l o g i s c h e s ~ I n s t i t u t, ~ U n i v e r s i t a ̈ t ~ B e r n, ~ C H-3012 ~ B e r n, ~ S w i t z e r l a n d, ~ a n d ~ '{ }^{3}$ Center for \\ Neural Science and Courant Institute of Mathematical Sciences, New York University, New York, New York 10003
}

Spontaneous episodic activity is a general feature of developing neural networks. In the chick spinal cord, the activity comprises episodes of rhythmic discharge (duration 5-90 sec; cycle rate $0.1-2 \mathrm{~Hz}$ ) that recur every 2-30 min. The activity does not depend on specialized connectivity or intrinsic bursting neurons and is generated by a network of functionally excitatory connections. Here, we develop an idealized, qualitative model of a homogeneous, excitatory recurrent network that could account for the multiple time-scale spontaneous activity in the embryonic chick spinal cord. We show that cycling can arise from the interplay between excitatory connectivity and fast synaptic depression. The slow episodic behavior is attributable to a slow activity-dependent network depression that is modeled either as a modulation of cellular excitability or as synaptic depression. Although the two descriptions share many features, the model with a slow synaptic depression accounts better for the experimental observations during blockade of excitatory synapses.

Key words: spontaneous activity; oscillations; depression; developing spinal network; recurrent excitation; rate model
Spontaneous activity generated by networks of synaptic connections is a characteristic, but poorly understood, feature of the developing nervous system. It has been detected in many different regions, including the retina, the hippocampus, and the spinal cord. In each region, bouts of activity occur episodically, separated by periods of quiescence. Although the mechanisms responsible for this activity are incompletely understood, it is known that developing networks are hyperexcitable, in part, because the classically inhibitory neurotransmitters, GABA and glycine, are depolarizing (Cherubini et al., 1991; Sernagor et al., 1995).

We have been studying this activity in the isolated lumbosacral spinal cord of the chick embryo. This preparation generates spontaneous episodes of activity in which spinal neurons are cyclically activated (see Fig. 1). This activity is not produced at a specific location, nor by a specific set of connexions (Ho and O'Donovan, 1993), and is still present in networks for which glutamatergic transmission or glycinergic/GABAergic transmission has been blocked (Chub and O'Donovan, 1998a). It has also been shown that episodes of activity transiently depress network excitability, which recovers in the quiescent phase; this depression is manifest as decreased synaptic strength (Fedirchuk et al., 1999) and hyperpolarization of individual cells. These observations have led to the proposal that the occurrence of episodes is a population phenomenon that is controlled by activity-dependent network depression (O’Donovan and Chub, 1997; O’Donovan, 1999). There is evidence that spontaneous bursts or episodes in

\footnotetext{
Received Aug. 31, 1999; revised Jan. 1, 2000; accepted Jan. 10, 2000.

J.R. acknowledges the support and hospitality of the Mathematical Research Branch, National Institute of Diabetes and Digestive and Kidney Diseases, and the Laboratory of Neural Control, National Institute of Neurological Diseases and Stroke, which facilitated his participation in this research. We thank Uri Cohen for comments on a previous version of this manuscript.

Correspondence should be addressed to Joel Tabak, Laboratory of Neural Control, Room 3A50, Building 49, National Institutes of Health, Bethesda, MD 20892. E-mail: joel@spine.ninds.nih.gov.

Copyright (c) 2000 Society for Neuroscience $\quad 0270-6474 / 00 / 203041-16 \$ 15.00 / 0$
}

other parts of the nervous system are also regulated by a form of activity-dependent depression. A slow "refractoriness" is thought to restrict wave generation and propagation in the newborn ferret retina (Feller et al., 1996, 1997; Butts et al., 1999). In hippocampal networks, NMDA receptor desensitization (Traub et al., 1994) or presynaptic depletion of glutamate pools (Staley et al., 1998) have been proposed to regulate the occurrence and/or duration of synchronous bursts.

Less is understood about the mechanism of cycling within an episode, but it has been proposed that this could also be regulated by activity-dependent depression with much faster kinetics than the depression controlling episodes [O'Donovan and Chub (1997); see also Streit (1993)]. Recently, it has been shown in modeling studies that a purely excitatory network with random connections susceptible to fast depression is capable of generating oscillations similar to the cycling observed during episodes (Senn et al., 1996; J. Streit and W. Senn, unpublished results).

Using these ideas, we develop a simple and general model of network activity. This model describes only the average firing rate of the population, rather than the membrane potential and spikes of the individual neurons. It assumes a purely excitatory recurrent network that is susceptible to both short- and long-term activity-dependent depression (see Fig. $2 A$ ). The model is described by only three coupled nonlinear differential equations. Therefore, the analysis can be done graphically, step by step, which facilitates an intuitive understanding of the network dynamics.

We show that this simple model can account for the occurrence of spontaneous episodes, the rhythmic cycling within an episode, and the developmental changes in the duration of episodes and interepisode intervals. Most importantly, it shows that the recovery of spontaneous activity in the presence of excitatory amino acid blockers (Barry and O'Donovan 1987; Chub and O'Donovan 1998a) is a property of networks with a slow form of synaptic depression. 

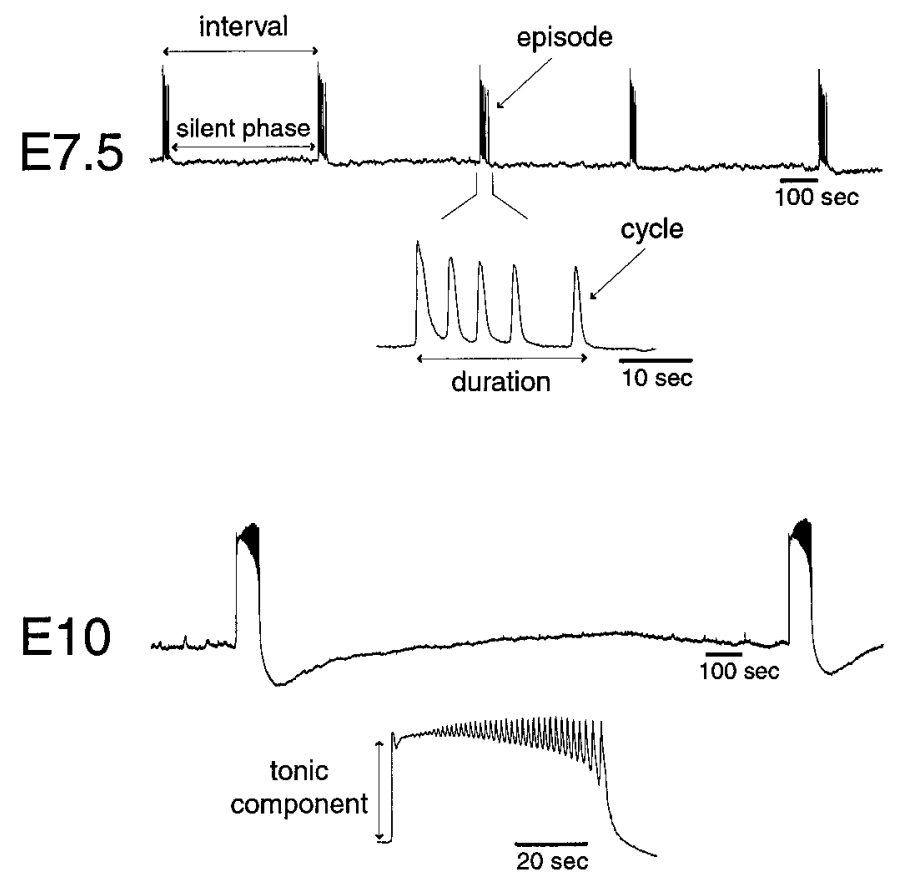

Figure 1. Spontaneous episodes of activity recorded from ventral roots of chick embryo spinal cord in vitro at embryonic day (E) 7.5 and 10. Recordings were made in DC mode and digitized at $10 \mathrm{~Hz}$, showing the synaptic depolarization recorded electrotonically from motoneurons. $\mathrm{Pe}$ riods of activity (episodes) are clearly distinct from silent phases. An interval is defined as the time between the beginning of two consecutives episodes. Episodes are composed of cycles, or "network spikes." With development, both the interval and episode duration lengthen, and cycle frequency and the level of depolarization (tonic component) also increase. Also, on older embryos episodes have a leading tonic phase before cycling begins. For the examples shown here, interval and episode durations are, respectively, E 7.5: $440 \pm 29$ and $20 \pm 1 \mathrm{sec}$; E 10: $1600 \pm 140$ and $68 \pm 3.5 \mathrm{sec}($ mean $\pm \mathrm{SD})$.

Parts of this work have been presented previously in abstract form (Rinzel and O'Donovan, 1997) and conference proceedings (O’Donovan et al., 1998; Tabak et al., 1999).

\section{MATERIALS AND METHODS}

\section{Experimental}

All experiments were performed on the isolated spinal cord of White Leghorn chicken embryos maintained in a forced-draught incubator. The spinal cord was dissected out under cooled $\left(12-15^{\circ} \mathrm{C}\right)$ oxygenated $(95 \%$ $\mathrm{O}_{2}, 5 \% \mathrm{CO}_{2}$ ) Tyrode's solution containing (in $\mathrm{mm}$ ): $139 \mathrm{NaCl}, 12$ glucose, $17 \mathrm{NaHCO}_{3}, 2.9 \mathrm{KCl}, 1 \mathrm{MgCl}_{2}, 3 \mathrm{CaCl}_{2}$. The preparation was transferred to a recording chamber (at room temperature) and superfused with oxygenated Tyrode's solution that was later heated to 26$28^{\circ} \mathrm{C}$. The concentration of $\mathrm{KCl}$ was raised to $5-8 \mathrm{~mm}$ during some of the recordings to accelerate the recovery of activity after glutamatergic blockade (Chub and O'Donovan, 1998a).

Neural activity was recorded from ventral roots or muscle nerves. Recordings were made using tight-fitting suction electrodes and amplified (DC $3 \mathrm{kHz}$ ) with high gain DC amplifiers (DAM 50 and IsoDAM, World Precision Instruments). Amplified signals were directly digitized through a PCI board (National Instruments) and/or recorded on tape (Neurodata) for further analysis. Recordings were digitized at a low sampling rate $(5-20 \mathrm{~Hz})$ because the events of interest occur on a slow time scale (Fig. 1).

\section{Theoretical}

Model equations. The two models are described and analyzed in depth in Results. Here we present them and the associated parameter values more succinctly. Both models have three dynamical variables, including $a$ and $d: a$ is the population activity or mean firing rate, and $d$ is the fast depression variable, representing the fraction of synapses not affected by the fast synaptic depression. These two variables generate the cycles within an episode. The slow modulation that underlies onset and termination of episodes, and the long silent phases, is described by $\theta$ or $s$. $\theta$ represents the threshold for cell firing. In the $\theta$-model, it increases slowly during an episode, eventually terminating the episode and recovering between episodes. In the $s$-model, $s$ represents the fraction of synapses not affected by the slow synaptic depression, decreasing slowly during an episode, terminating it, and then recovering between episodes. In the $s$-model, the fraction of nondepressed synapses at any given time is therefore the product $s \cdot d$.

The equations for the models are:

$\theta$-model:

$$
\begin{aligned}
\tau_{\mathrm{a}} \dot{a} & =a_{\infty}(n \cdot d \cdot a)-a, \\
\tau_{\mathrm{d}} \dot{d} & =d_{\infty}(a)-d, \\
\tau_{\theta} \dot{\theta} & =\theta_{\infty}(a)-\theta,
\end{aligned}
$$

$s$-model:

$$
\begin{aligned}
& \tau_{\mathrm{a}} \dot{a}=a_{\infty}(n \cdot s \cdot d \cdot a)-a, \\
& \tau_{\mathrm{d}} \dot{d}=d_{\infty}(a)-d, \\
& \tau_{\mathrm{s}} \dot{s}=s_{\infty}(a)-s .
\end{aligned}
$$

The parameter $n$ measures the connectivity in the network (see Results). All of the $x_{\infty}$ functions are sigmoidal (e.g., see Fig. $2 C$ for a plot of $\left.a_{\infty}\right)$ :

$$
\begin{gathered}
a_{\infty}\left(I_{\mathrm{eff}}\right)=1 /\left(1+e^{-\left(\mathrm{I}_{\mathrm{eff}}-\theta\right) / \mathrm{k}_{\mathrm{a}}}\right) \\
d_{\infty}(a)=1 /\left(1+e^{\left(\mathrm{a}-\theta_{\mathrm{d}}\right) / \mathrm{k}_{\mathrm{d}}}\right) \\
\theta_{\infty}(a)=1 /\left(1+e^{-\left(\mathrm{a}-\theta_{\theta}\right) / \mathrm{k}_{\theta}}\right) \\
s_{\infty}(a)=1 /\left(1+e^{\left(\mathrm{a}-\theta_{\mathrm{s}}\right) / \mathrm{k}_{\mathrm{s}}}\right) .
\end{gathered}
$$

The parameter $\theta_{\mathrm{x}}$ defines the argument's value for which half-activation occurs: $x_{\infty}\left(\theta_{\mathrm{x}}\right)=1 / 2$. The value of $k_{\mathrm{x}}$ (positive) determines the sigmoid's steepness, smaller values being steeper. Note that the presence (respectively absence) of a minus sign in front of $\left(a-\theta_{\mathrm{x}}\right)$ indicates that the sigmoid is increasing (respectively decreasing). The variables $d$ and $s$ are high for low activity (low depression) and low for high activity (high depression), so $d_{\infty}(a)$ and $s_{\infty}(a)$ are decreasing functions of $a$.

Although $a_{\infty}$ is the cellular input-output function, it should be considered as an average over the population of neurons. Assuming these neurons are not identical, $a_{\infty}$ inherits a sigmoid shape; $\theta$ can be interpreted as the mean threshold and $k_{\mathrm{a}}$ as the threshold variance, assuming a unimodal distribution of thresholds (Wilson and Cowan, 1972). Because the connectivity coefficients are included inside the $a_{\infty}$ function, $a$ should be interpreted as mean population firing rate averaged over a brief period of time, not as instantaneous voltage (Pinto et al., 1996); $a_{\infty}(n \cdot s \cdot d \cdot a)$ is the instantaneous firing rate. Note that because $a_{\infty}(0)$ is not equal to zero, we are implicitly assuming that there is some background activity or fraction of spontaneously active neurons in the network. However, the model does not depend on this feature. Indeed, if $a_{\infty}(0)=$ 0 , similar episodes of activity are produced, except that $a$ stays virtually at 0 from just after an episode to just before the next one (our unpublished results).

Unless stated otherwise, we use the parameter values given in Table 1. Simulations. The model equations were implemented within XPPAUT (freely available software by G. B. Ermentrout, http://www.pitt.edu/ phase/), a general purpose interactive package for numerically solving and analyzing differential equations. XPPAUT includes a tool for calculation of bifurcation diagrams (AUTO). Simulations were performed using the Runge-Kutta integration method with a time step of 0.2 (dimensionless units). We checked that the results were unchanged when the time step was halved. Simulations were run on Unix PCs. A version of the software WinPP runs under Win95 or Win98.

\section{RESULTS}

We have argued elsewhere (Ritter et al., 1999) that the spinal network responsible for spontaneous activity is composed of 


\begin{tabular}{lcc}
\hline $\begin{array}{l}\text { Table 1. Values of the parameters used for each model, unless } \\
\text { mentioned otherwise in text or figure }\end{array}$ \\
Parameter & $\theta$-model & $s$-model \\
\hline$n$ & 1 & 1 \\
$\tau_{\mathrm{a}}$ & 1 & 1 \\
$\theta$ & Variable \\
$k_{\mathrm{a}}$ & 0.05 & 0.18 \\
$\tau_{\mathrm{d}}$ & 2 & 0.05 \\
$\theta_{\mathrm{d}}$ & 0.2 & 2 \\
$k_{\mathrm{d}}$ & 0.5 & 0.5 \\
$\tau_{\theta}$ & 1000 & 0.2 \\
$\theta_{\theta}$ & 0.15 & \\
$k_{\theta}$ & 0.05 & \\
$\tau_{\mathrm{s}}$ & & 500 \\
$\theta_{\mathrm{s}}$ & & 0.14 \\
$k_{\mathrm{s}}$ & & 0.02 \\
\hline
\end{tabular}

recurrently connected, functionally excitatory synaptic connections. Furthermore, because the expression of network activity does not appear to depend on the details of network architecture or membrane properties and because detailed biophysical information on cell and coupling properties is unavailable, we are justified in using a very idealized model. Throughout, we use mean field models that describe only the averaged population behavior, not the detailed electrical activity of individual cells. The models are also temporally coarse-grained so that action potentials are not represented per se, only firing rate.

Our graphical analysis of the models proceeds by considering reduced models, beginning with the fastest process first and expanding the model by stepwise introduction of the next slower variables. We begin with the single Equation 1 that governs the dynamics of a recurrent excitatory network, without the negative feedback of depression. This reduced model illustrates the classical behavior of bistability in a recurrent population, of either a low or high activity state of steady firing. Next, we introduce a fast activity-dependent synaptic depression, which can lead to network oscillations (cycling) in this two-variable system. We analyze this subsystem with phase plane methods, finding a different type of bistability. Finally, we add slow activity-dependent depression in either of two forms and show that these three-variable models can generate episodes similar to those observed experimentally in the chick embryo spinal cord and other networks.

\section{A recurrent excitatory network can be bistable}

In this section, we consider only the dynamics of the average activity in a random excitatory network; the depression variables are frozen. We use a graphical method to illustrate how the steady activity states of the one-variable system are determined. These steady states depend on parameters such as the network connectivity, and we show that for certain values of connectivity the network can have two stable states.

Here, and throughout, we let $a$ be a measure of the network activity, for instance the population's mean firing rate, relative to the maximum possible rate. Its time behavior, according to classical descriptions (Wilson and Cowan, 1972), satisfies $\tau_{\mathrm{a}} \dot{a}=$ $a_{\infty}\left(I_{\text {eff }}\right)-a$, where $\dot{a}$ is the time derivative of $a$ and $I_{\text {eff }}$ is the effective input to cells in the network. Accordingly, the activity of the network reaches the value $a_{\infty}\left(I_{\text {eff }}\right)$ with a time constant $\tau_{\mathrm{a}} \cdot a_{\infty}$ is called the activation function and describes how $I_{\text {eff }}$ is converted into firing rate (Fig. $2 B$ ). For small inputs, $a_{\infty}$ is near 0 ;
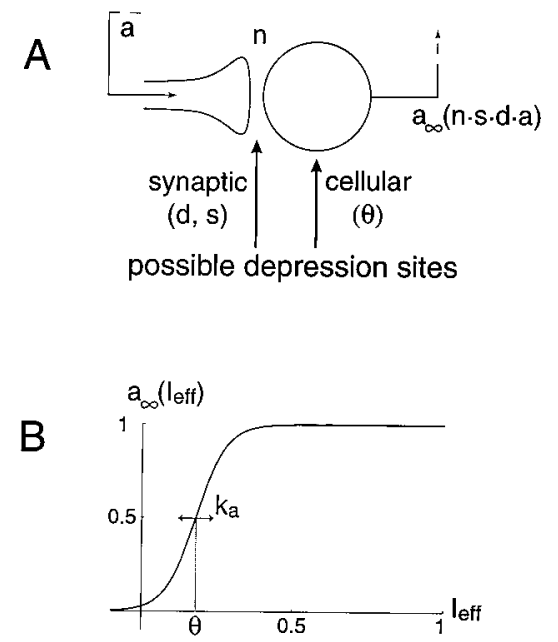

Figure 2. $A$, Schematic diagram of recurrent network with random connections. The output from the network is fed back to the cells. The amount of feedback depends on network connectivity $(n)$. Activity $(a)$ can also be modulated at the synaptic level by a fast depression $(d)$ and by a slow depression $(s)$. The effective input to cells is therefore $n \cdot s \cdot d \cdot a$. The resulting postsynaptic firing rate, at steady state, is given by $a_{\infty}(a \cdot d \cdot s)$. This postsynaptic firing rate can also be modulated by the cells' threshold $(\theta)$. Note, our mathematical formulation does not distinguish presynaptic and postsynaptic effects. $B$, Cellular input-output relationship, averaged over the population. $I_{\text {eff }}$ is the effective input to the network (see Results). The cellular threshold parameter $\theta$ can also be viewed as a slowly modulated variable. $k_{\mathrm{a}}$ measures the width of the transition range around threshold; a small value of $k_{\mathrm{a}}$ implies a steep activation function.

very few neurons are firing. For large inputs, $a_{\infty}$ saturates at 1 , with all cells in the network being fully active. There is a rather sharp transition between these two limits (if $k_{\mathrm{a}}$ is small) at the value $I_{\text {eff }}=\theta$ for which $a_{\infty}(\theta)=1 / 2$. $\theta$ corresponds to the average threshold for cell firing. The mathematical expression of $a_{\infty}$ is given in Materials and Methods.

Because of the recurrent connectivity, the input $I_{\text {eff }}$ is produced by the network's own activity, in addition to any external inputs. Therefore, in the absence of external inputs $I_{\text {eff }}=n \cdot a$, where we introduce the parameter $n$ as a measure of network connectivity (in arbitrary units). The greater the connectivity, the greater the recurrent input being fed back to the network for a given amount of activity ( $n$ is a gain parameter, measuring how the average cellular output is transmitted to other cells, and thus depends on many physiological parameters such as the average number of contacts from a neuron, average synaptic efficacy, etc.). Thus, the dynamics of this network is described by:

$$
\tau_{\mathrm{a}} \dot{a}=a_{\infty}(n \cdot a)-a .
$$

What is the behavior of the system defined by Equation 1'? After any transient input, $a$ will evolve to a steady state, for which $\dot{a}=0$. Its value at steady state must then satisfy $a=a_{\infty}(n \cdot a)$. The solutions to this equation are represented graphically in Figure $3 A$ as the intersections of the curves $f(a)=a$ and $g(a)=a_{\infty}(n \cdot a)$ for different values of $n$.

For small values of $n$, there is only one steady state, at a low value of $a$, with the network barely active because of its low, functional connectivity. Even if the network receives a strong transient external input, it will return to this quiescent state because the connectivity is too low to sustain the activity. Similarly, for large connectivity, there is only one possible steady state (the curves do not actually intersect at the low level), for which 

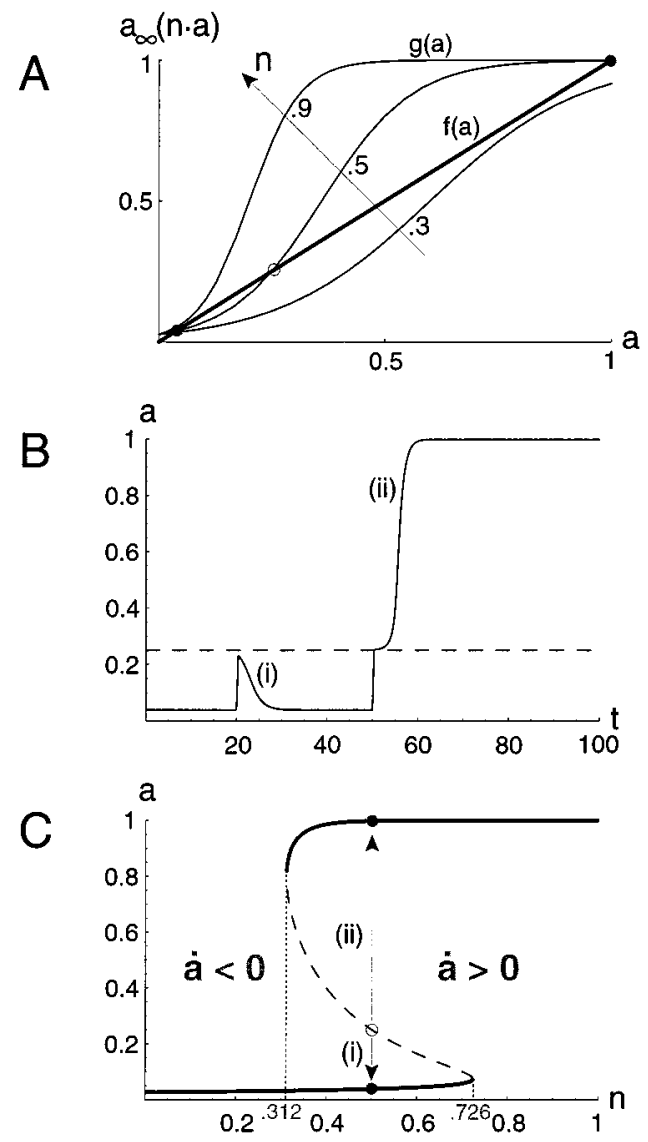

Figure 3. Analysis of the single-variable activity model for a recurrent excitatory network. $A$, The steady states satisfy $a_{\infty}(n \cdot a)-a=0$. They are determined by the intersection(s) between the curves $f(a)=a$ (thick line) and $g(a)=a_{\infty}(n \cdot a)$ (smooth curves), shown here for different values of $n$. At these intersections $a_{\infty}(n \cdot a)-a=0 . B$, Time course of the activity for $n=0.5$. At $t=20$ a brief stimulus is applied but fails to bring the network above threshold $(i)$. Stronger stimulation applied at $t=50$ excites the network above threshold, so the high activity steady state is reached (ii). The network will stay at this high level unless a large perturbation brings it back below threshold. $C$, Plot of the steady-state values of $a$ for all possible values of $n$ between 0 and 1 . The dashed portion of the curve indicates unstable steady states. In $A$ and $C$, the steady states for $n=0.5$ are represented by filled (stable) or open (unstable) circles. The arrows indicate that the activity, if initially below threshold (unstable steady state), will fall back to the low stable state $(i, \dot{a}<0)$, and if it is initially above network threshold it will increase until it reaches the high stable state $(i i, \dot{a}>0)$, as illustrated in $B . \theta=0.18$.

the network is very active ( $a$ near 1$)$. A more interesting case occurs for intermediate values of the connectivity parameter: there are three steady states, at low, intermediate, and high values of $a$. However, the intermediate steady state is unstable. That is, a small perturbation from it will grow, and the network activity will evolve toward one of the two other steady states. Therefore, the network has two possible stable steady states; it is bistable. If the network is in the low steady state, it can be switched to the high steady state by a transient stimulus that brings $a$ above the intermediate steady state, which is the effective network threshold. If the stimulation does not increase the activity above threshold, the network will fall back to low activity after the stimulus. This network threshold is not equal to the cell threshold $\theta$; for example, if $a_{\infty}$ is very steep, the network threshold approximately equals $\theta / n$; it decreases with increasing connectivity. Note that once the network has switched from the low to high (or high to low) state, it will stay in the new state even though the perturbation has stopped. It will not return to the previous state, unless a new perturbation of sufficient magnitude occurs (if neural noise were incorporated explicitly in the model there might be spontaneous transitions between the two states). This type of bistable behavior, illustrated in Figure $3 B$ (see figure legend), is characteristic of recurrent excitatory networks (Wilson and Cowan, 1972). In the next sections, we will find that bistability underlies our models' episodic rhythms; slow modulation leads to transitions between the attracting pseudostates of cycling during an episode and quiescence between episodes.

Figure $3 C$ summarizes the above results by showing the activity levels at steady state ( $\dot{a}=0)$ over a range of $n$ values. Again, it illustrates that for low connectivity/efficacy ( $n$ small) there is only one steady state at low activity. For strong connectivity ( $n$ near 1 and above) only the high activity state is present. For an intermediate range of $n$ values both stable states coexist, along with an unstable state of intermediate activity (dashed line). The states are continuously connected as you travel along the S-shaped curve. However, as $n$ is varied over the range, one encounters discontinuous transitions between the stable states, at the S-curve's knees. This figure, showing the different possible activity states for all values of a control parameter, is called a bifurcation diagram [see Strogatz (1994); a bifurcation is a point for which there is a qualitative change in the dynamics of the system, like at the knees]. It will be useful to distinguish the three "branches" of such S-curves: upper, lower, and middle. Here, upper and lower ones correspond to the stable steady states, and the middle one corresponds to the unstable steady state. The middle branch is a separatrix between the upper and lower branches: any state of the network above it (i.e., on the right side of the S-curve) will evolve toward the high steady state, and any state below it (i.e., on the left side of the diagram) will fall to the low steady state. It is easy to understand why: for any point on the S-curve, by definition, $\dot{a}=0$. If the connectivity is increased (i.e., the point shifted to the right) even slightly, this increases $a_{\infty}(n \cdot a)$, and therefore $\dot{a}>0$. Conversely, if $n$ is decreased this leads to $\dot{a}<0$.

\section{A recurrent excitatory network with fast activity-dependent synaptic depression can produce oscillations}

In the above section, we studied the behavior of a recurrent excitatory network as a function of its (fixed) connectivity. Here, we will analyze network behavior when the effective connectivity is changing because of synaptic depression. We will analyze the resulting two-variable system graphically in the phase plane and show that it can generate oscillations (like the cycling during an episode) and bistability, depending on the average neuronal excitability.

As a preliminary step, reconsider Figure $3 C$ and suppose that we sweep $n$ slowly back and forth over a range that includes the bistable regime. This would lead to alternating phases of high and low activity, with sharp transitions between phases. If $n$ were decreasing while the activity is high, the system's state point would track the upper branch, moving leftward, until we reached the S-curve's left knee. Then the state point would fall to the lower branch. The activity would drop precipitously. Now at low activity, if $n$ were increasing, the system would track the lower branch, with activity rising very gradually, until the right knee was reached. Then the state point would jump back to the upper branch of high activity, completing a cycle, and the process would repeat. 
We really want a description so that such cycling happens spontaneously, rather than being driven as above. With this goal we make the effective connectivity a dynamic variable by multiplying $n$ by the variable $d$, the kinetics of which depends on activity, guaranteeing that $d$ automatically decreases when activity is high and increases for low activity. In this formulation, $d$ is defined as the fraction of nondepressed synapses from a representative cell to its targets (e.g., the fraction of releasable transmitter at those synapses). Its value ranges from 0 (synapses totally depressed) to 1 (all synapses fully available), so the effective connectivity $(n \cdot d)$ ranges from 0 to $n(n$, being an arbitrary measure of connectivity can be $>1$ ). In all of the following figures, we set $n=1$ unless mentioned otherwise. Equation $1^{\prime}$ thus becomes:

$$
\tau_{\mathrm{a}} \dot{a}=a_{\infty}(n \cdot d \cdot a)-a .
$$

This idealized model does not distinguish between glutamatergic excitatory synapses or depolarizing gabaergic/glycinergic synapses.

We use a simple first-order kinetics for $d$, reminiscent of the gating kinetics in Hodgkin-Huxley-like models for voltage-gated ionic currents:

$$
\tau_{\mathrm{d}} \dot{d}=d_{\infty}(a)-d
$$

where $d_{\infty}(a)$ is a decreasing sigmoid function (see Materials and Methods). When the system is at low activity, $d$ tends to increase, whereas for high activity $d$ decreases. $d$ tends toward $d_{\infty}(a)$ but lags behind changes in $a$ with a time constant $\tau_{\mathrm{d}}$. Hence, the system can potentially oscillate, but whether it does depends on parameter values. For example, if the negative feedback from depression is too fast, the oscillations are precluded. The condition (parameter relationship) for which oscillations emerge in the model is given in the Appendix.

We need to examine the dynamics of this $a-d$ system over a range of $\theta$ values, because in later sections $\theta$ will become a slow modulatory variable. For $\theta$ small, the network is highly excitable, and it operates at a steady high-activity level. If the network is held at a low activity level with synapses fully available and then released (Fig. 4A), it tends to its desired high level; in this case, it shows damped oscillations around the steady state because the depression is delayed (in the same way as the "delayed rectifier" potassium current does not turn on immediately after a voltage increase).

The system's time course from Figure $4 A$ can be viewed in another way, as a trajectory by plotting in the $(a, d)$ plane simultaneously the points defined by the values of $a$ and $d$ at consecutive and equally spaced times (Fig. $4 B$, dotted line). Although time is not represented explicitly on such a diagram, velocity along the trajectory can be deduced from the spacing between consecutive points. Here, on release from low activity the network quickly (large spacing between points) moves toward a state of high activity with the synapses depressed, slowing down (small spacing between points) as it approaches the steady state, in a spiraling fashion (the damped oscillation).

Using the $a-d$ plane, we can analyze and predict qualitatively the system's dynamical behavior and the consequences of changing various parameters [for an introduction to phase plane analysis, see Rinzel and Ermentrout (1998) or Strogatz (1994)]. The power of this graphical/geometrical approach comes largely from understanding the two solid curves in Figure $4 B$. These curves are called the nullclines. The $a$-nullcline is defined as the relationship between $a$ and $d$ that is obtained by setting $\dot{a}=0$. Similarly, the
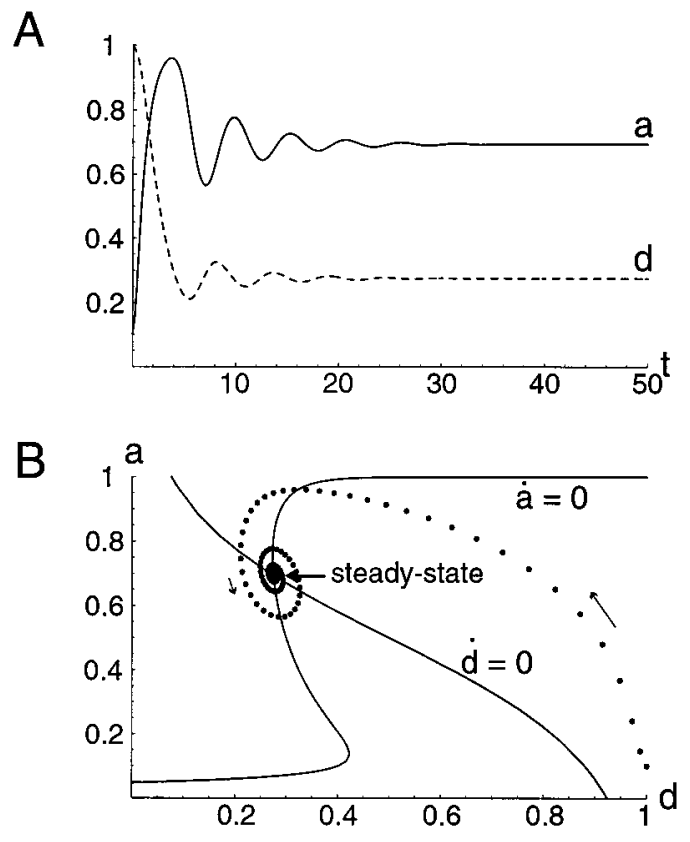

Figure 4. Dynamical behavior of the $a-d$ system for low firing threshold, $\theta(0.15)$. $A$, Time course of activity $a$ (solid curve) and depression $d$ (dashed curve) showing that the system reaches a steady state after some damped oscillations. B, Phase-plane representation of the dynamics, showing the nullclines and trajectory of network state. The $a$ - and $d$-nullclines are defined by $\dot{a}=0$ and $\dot{d}=0$. There is only one intersection, therefore the system has only one steady state. It is a stable steady state; as shown by the time course $(A)$ or phase-plane trajectory. The damped oscillations evident in the time course $(A)$ are seen here as spiralling toward steady state. $n=1$.

$d$-nullcline comes from setting $\dot{d}=0$. Given these nullclines, we can map out the flow directions in the phase plane, according to the different areas delimited by the nullclines. For example, in the area on the right of the $a$-nullcline and above the $d$-nullcline, we know that $a$ must be increasing and $d$ decreasing (hence the arrow pointing up and left). Also, we know that $a$ must be at a local minimum or maximum when the trajectory crosses the $a$-nullcline and so on. Where the nullclines intersect, neither $a$ nor $d$ is changing, and this corresponds to a steady state of the system.

The $a$-nullcline is defined implicitly according to $a=a_{\infty}(n \cdot d \cdot a)$. For a physiological interpretation, consider $d$ as a parameter so that points on the $a$-nullcline are the steady-state values of $a$ at which decay of $a$ balances the regenerative input from recurrent excitation in Equation 1. Of course, the result is similar to the S-curve in Figure $3 C$. The $d$-nullcline is simply the graph of $d_{\infty}(a)$. The nullclines are drawn in Figure $4 B$, showing that there is only one possible steady state (the unique intersection of the two curves). In general there can be one or three (exceptionally two) intersections, as we will show below for some values of $\theta$. The $a$-nullcline has the characteristic switchback form (S-shape) of autocatalysis in nonlinear excitable and oscillatory systems. Although the steady state is stable in this case, it could be made unstable by increasing $\tau_{\mathrm{d}}$ to slow the negative feedback process, in which case the activity would become oscillatory.

For sufficiently large values of $\theta$, there is again only one steady state, but now at a low activity level: in Figure $5 B$, the unique intersection of the nullclines near the abscissa at $d=0.923$ (vertical arrow). Note that the $a$-nullcline's low-activity branch is very close to the abscissa. This network model is excitable. If the system is stimulated briefly but adequately while at this quiescent, 

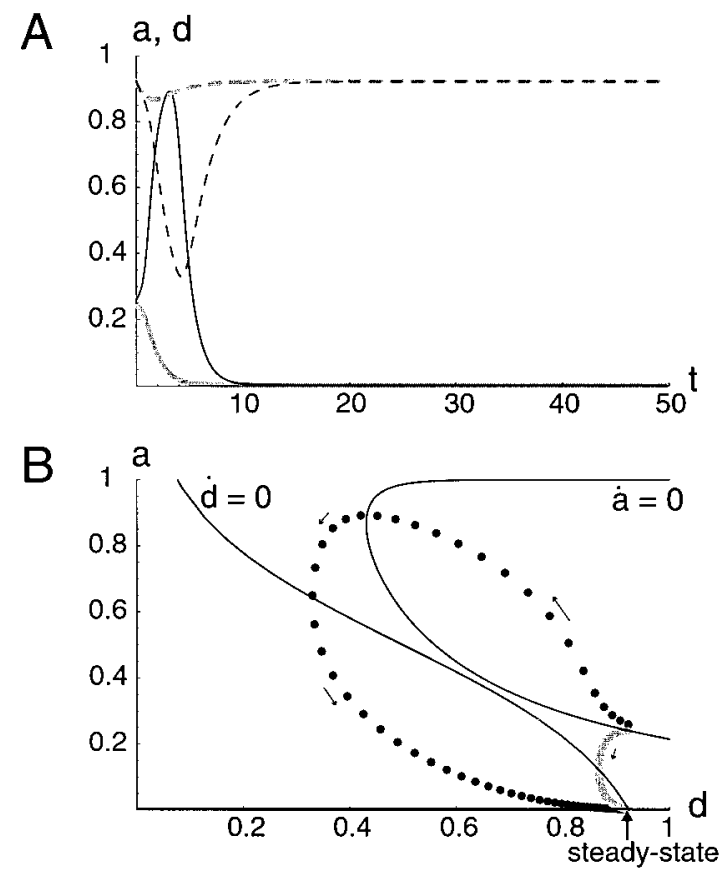

Figure 5. Dynamical behavior of the $a-d$ system for high $\theta(0.28)$. A, Time course for two different initial conditions. In one case, $a(0)$ is above a threshold, and the network exhibits a transient high-activity phase and then falls to quiescence (black curves). In the other case, the network goes directly to "rest" (gray curves). $a$ is indicated by the solid curves; dashed curves represent $d$. $B$, Phase-plane representation of the dynamics, showing trajectories for the two cases illustrated in $A$. There is only one steady state, defined by the intersection of the $d$-nullcline and the lower branch of the $a$-nullcline (for $a \simeq 0$ )-the quiescent state. If the system is perturbed away from steady state by a sudden increase in activity, the threshold for generation of a transient cycle of high activity is approximately given by the middle branch of the $a$-nullcline (it is actually slightly above). $n=1$.

resting state, it exhibits a transient response of high activity and then returns to rest. Figure $5 A$ shows the threshold effect by plotting $a(t)$ and $d(t)$ for two different initial conditions. In one case (gray curves), the activity starts just below threshold and directly returns to the steady state. In the other case (black curves), the activity is initially just above threshold and then increases in a regenerative way before depression returns it to the steady state. Note that this threshold for network excitability is approximately given by the value of the middle branch of the $a$-nullcline and should not be confused with $\theta$, which is the average cellular threshold. This phase plane portrait gives us a graphical way to see the effect of increased threshold, by comparison with Figure $4 B$. The $a$-nullcline's right shift is understandable intuitively. For a given value of depression variable $d$, one expects with increasing $\theta$ to lose the possibility of a high-activity state in the $a$-subsystem. With enough increase of $\theta$ the highactivity state in the full $a-d$ system is precluded.

Finally, for some intermediate values of $\theta$, there can be three steady states, as shown on Figure 6, $A$ and $B$. In both $A$ and $B$, the lower steady state is stable, and the intermediate one (very close to the low state) is unstable. However, the stability of the highlevel steady state depends on the particular values of the other parameters. An important parameter, which does not change the location of the steady states, is $\tau_{\mathrm{d}}$. The high-level steady state is stable in Figure $6 A$, for a value of $\tau_{\mathrm{d}}=1$. Hence, the system is bistable. If the value of $\tau_{\mathrm{d}}$ is increased, the negative feedback
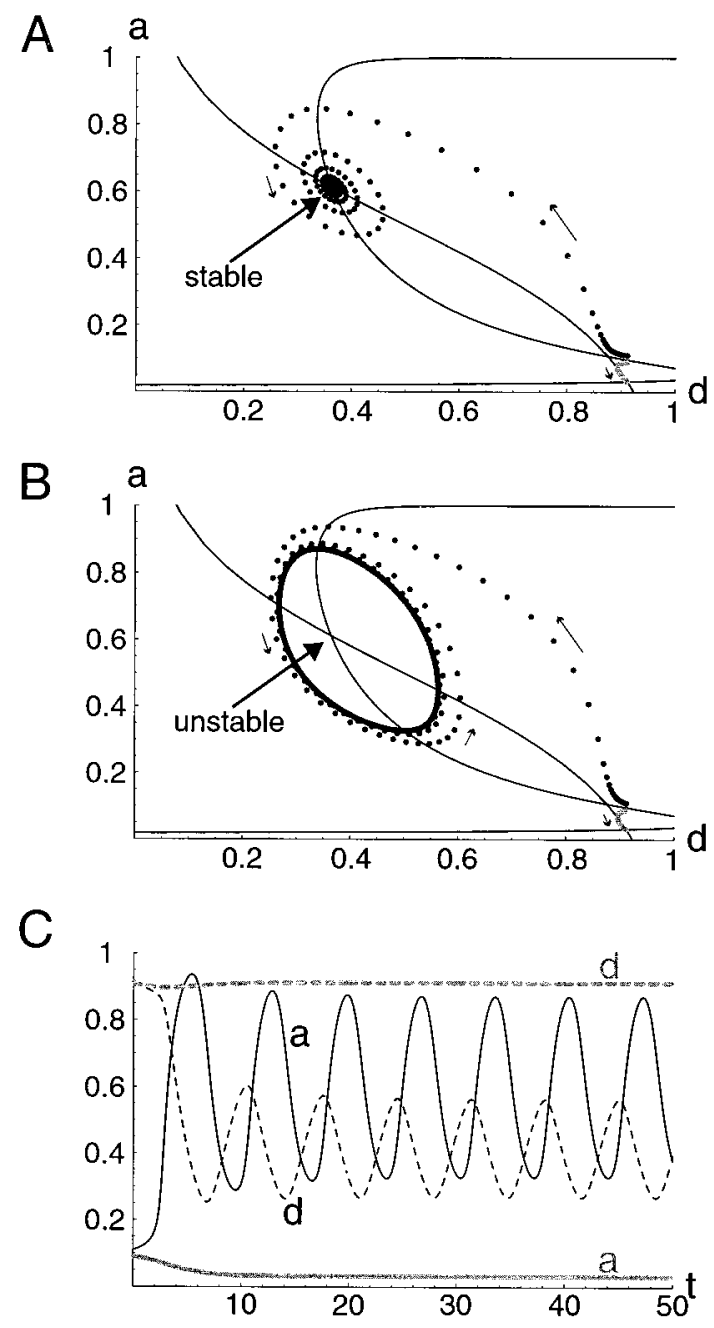

Figure 6. Dynamical behavior of $a-d$ system for intermediate $\theta(0.2)$. $A$, Phase-plane representation of the dynamics for $\tau_{\mathrm{d}}=1$. The high and low steady states are stable. Any trajectory eventually reaches one of them, depending on initial conditions. A perturbation away from the low steady state can switch the system to the high state, if it is above the middle branch (black trajectory). Otherwise, the system falls back to the lower state (gray trajectory). B, Phase-plane representation of the dynamics for $\tau_{\mathrm{d}}=2$. This higher value destabilizes the high steady state and leads to the appearance of a stable oscillation (closed orbit) around it. Again, a sufficient perturbation can switch the system from the low-activity steady state to oscillations. $C$, Time courses for the trajectories shown in $B$. Superthreshold perturbation leads to an oscillation (black curves), whereas after a subthreshold perturbation, activity returns directly to the low steady state (gray curves). The dashed curves represent $d . n=1$.

caused by depression is more delayed, which can lead to oscillations around the steady state (now unstable), as shown in Figure $6 B$. The system is bistable again, but now we have a low-activity steady state coexisting with a high-activity oscillatory state. Depending on the initial conditions (or external inputs), the system can either reach the low steady state or the high activity state, either steady (Fig. $6 A$ ) or oscillatory (Fig. 6B,C).

We summarize this collection of possible behaviors in a bifurcation diagram (Fig. 7A) by plotting the $a$-values for the steady and oscillatory states when the parameter $\theta$ is varied. The $\mathrm{Z}$-shaped curve (black solid line and dashes) for steady states is analogous to the S-curve of Figure $3 C$; the orientation is leftright-reversed because increasing $\theta$ reduces network excitability, 

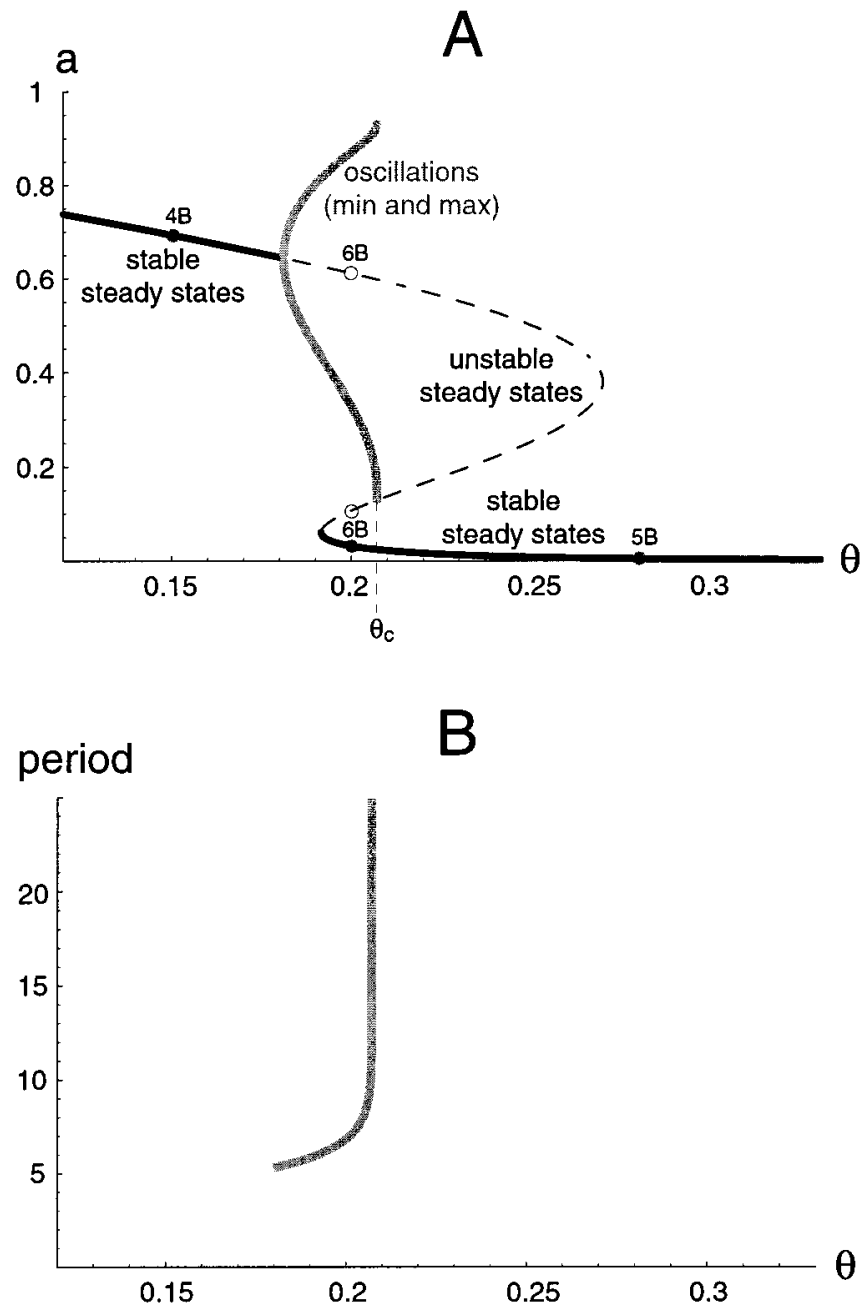

Figure 7. A, Bifurcation diagram showing the possible behaviors of the $a-d$ fast subsystem for different values of $\theta$. For low values of $\theta$, there is only one steady state, at high level, which becomes unstable for increasing $\theta$. For intermediate values of $\theta$ (around 0.2 ), the system is bistable: there is a stable high-activity oscillation and a stable, low steady state. With larger values of $\theta$, the system can only be in a low steady state. Circles represent the cases shown in Figures $4 B, 5 B$, and $6 B$. Thick black curves: stable steady states; dashed, thin curve: unstable steady states; thick gray curve: maximal and minimal values of $a$ for the periodic oscillations. $B$, Period of the oscillations.

like decreasing $n$. Starting at $\theta$ low with the unique high-activity steady state and moving rightward along this curve, we see that this state loses stability at $\theta>0.181$ (solid turns to dashed). At this point, a stable oscillation emerges, around the steady state. We plot the maximum and minimum values of $a$ during a cycle (thick gray curves). At emergence, the oscillation's amplitude is very low, but then grows as $\theta$ increases. This type of oscillation birth is called a Hopf bifurcation (Rinzel and Ermentrout, 1998). At a critical value of $\theta\left(\theta_{c}=0.207\right)$, the oscillation encounters the unstable state on the Z-curve's middle branch and disappears. That is, the minimum activity during a cycle is just equal to the excitation threshold, and the oscillation cannot be maintained if $\theta$ is increased any farther. For larger $\theta$ only the low-activity steady state is stable.

The system is bistable for $\theta$ between the Z-curve's left knee and $\theta_{\mathrm{c}}$. Over this range, it can be either at a low, steady activity level, or at a high, oscillatory level. These high-activity oscillations mimic the cycling observed during an episode of activity in the chick spinal cord. In the next sections we will see how slow autonomous dynamics for this modulatory variable $\theta$ can sweep the $a-d$ subsystem back and forth over this bistable regime to account for the cord's episodic rhythms. Thus, although Figure $7 A$ does not show motion per se, it forms the skeleton over which episodic dynamic patterns can be understood. For example, as shown from Figure $6 A, B$, the value of $\theta$ where the cycles emerge depends on parameters such as $\tau_{\mathrm{d}}$. If $\tau_{\mathrm{d}}$ is decreased, the oscillations emerge for higher values of $\theta$. Consequently, when an episode is started, the system first jumps toward a high stable steady state, then with increased $\theta$ this high state becomes unstable and oscillations emerge. Thus, our model when the slow variable is included may exhibit, depending on parameter values, episodes that have phases of near steady high-activity before oscillations start, as sometimes seen experimentally (Fig. 1, bottom panel).

Figure $7 B$ shows the variations of oscillation period with $\theta$. For the lowest possible value of $\theta$ allowing oscillations, the period is finite. The period lengthens as $\theta$ increases, first slowly and then abruptly just before the oscillations disappear (it becomes infinite at the exact point where the low oscillatory level meets the intermediate, unstable state). Except for the very end of the range, the cycle period ranges between 5 and 10 units of time (approximately 7 for the activity shown on Fig. 6C). Therefore, because the typical cycle period measured experimentally is $\sim 1$ sec for embryonic day (E) 10 embryos, $\tau_{\mathrm{d}}=2$ predicts that the time constant of the depression variable $d$ is of the order of 200-400 msec. This is comparable to time scales reported in other systems (Chance et al., 1998).

\section{Slow activity-dependent depression leads to episodic behavior}

In the chick embryo, after an episode of activity, evoked monosynaptic and polysynaptic potentials are depressed and recover with a time constant of $\sim 1$ min (Fedirchuk et al., 1999). In addition, after an episode, the membrane potential of ventrally located spinal neurons hyperpolarizes and subsequently recovers as a depolarizing ramp until the next episode. Collectively, these observations indicate the presence of a slow (time scale minutes) activity-dependent form of depression that regulates network excitability and has been proposed to control the occurrence of episodes (O'Donovan and Chub, 1997). Although the mechanisms for these changes are unknown, they could occur through a modulation of cellular properties or a prolonged "slow" form of synaptic depression. In the following, we formulate and analyze models for each of these two possibilities.

The two-variable system presented in the previous section models a bistable, recurrent excitatory network that can exhibit persistent states of low and high activity, the latter being oscillatory. In contrast, the chick's spinal cord shows spontaneous alternation between two such states. Here, we add a slow, third variable that enables the model to generate sustained, repetitive episodes of cycling at high activity separated by low-activity phases, thereby mimicking the spontaneous, episodic rhythm of the cord. We show that the mathematical structure that underlies the episodic behavior is the same for both types of slow activitydependent depression. Therefore, the two models behave in similar ways, say, when perturbed by a brief stimulation or noisy input, and they are affected similarly by some parameter variations. In a later section, however, we show that the models lead to 
different predictions for variations in connectivity and therefore the models can be distinguished experimentally.

\section{Slow modulation of cellular excitability: $\theta$-model}

Intracellular recordings have shown that there is a slow ramp depolarization in motoneurons of chick embryonic spinal cord (N. Chub and M. J. O’Donovan, unpublished observation), suggesting that after an episode the functional threshold $(\theta)$ of neurons is raised and slowly decreases until the next episode. We can therefore imagine that $\theta$ increases during an episode and decreases during the silent phase. This means that the growth and decay of $\theta$ is activity-dependent, analogous to a slow adaptation mechanism. During high activity the threshold rises, eventually terminating the activity, and then it recovers during the interepisode quiet phase. When the threshold decreases adequately, the network's small amount of spontaneous activity can trigger another episode. We formulate below a kinetic model for $\theta$ that yields this behavior.

From our dynamical systems viewpoint and the previous sections, we can predict the model system's behavior as follows (we will confirm this below in Figure 8 with a simulation). First, because $\theta$ will move very slowly, we expect that the $a-d$ subsystem will evolve relatively rapidly to one of its two attractors (Fig. 7A) and then track it as $\theta$ moves. Suppose the network is in the low-activity state, as after an episode. Under this condition $\theta$ will slowly decrease, and the $a-d$ subsystem will slowly track the Z-curve's lower branch until the left knee is reached. The system then rapidly moves to the oscillatory high-activity state, starting an episode. During the episode $\theta$ now increases, and as indicated by the thick gray curves on Figure $7 A$, the cycle amplitude also gradually increases. Moreover, the cycle period increases too (Fig. $7 B$ ), but only slightly at the beginning of the episode, then significantly near the end of the episode. Note, this significant drop in period just before episode termination is a generic feature of this type of model and is typical of many experiments in chick cord (Fig. 1, top panel). Eventually, $\theta$ reaches the critical level $\theta_{\mathrm{c}}$ where activity can only jump back to the lower steady state, terminating the episode. As $\theta$ then decreases again (recovery), the process will be repeated. Thus, the presence of bistability of the $a-d$ subsystem for a range of $\theta$ values suggests a mechanism for the slow occurrence of episodes, in the same way that the bistability of the simple recurrent network over a range of connectivity values suggested a mechanism for the generation of cycles during an episode. Our prediction of the slow rhythm's $a-\theta$ trajectory rests on the assumptions (i.e., on our construction) that (1) the fast $a-d$ subsystem is bistable, (2) $\theta$ evolves very slowly, and (3) $\theta$ evolves in the proper directions during the active and quiescent phases. These features form the essence of the fast/slow dissection technique for analyzing multiple time scale oscillations and excitability of this sort (Rinzel and Ermentrout, 1998).

As for $a$ and $d$, we model the variations of $\theta$ with a simple, first-order kinetics:

$$
\tau_{\theta} \dot{\theta}=\theta_{\infty}(a)-\theta
$$

with $\theta_{\infty}$ an increasing (sigmoidal) function of $a$ and $\tau_{\theta}$ being two to three orders of magnitude greater than $\tau_{\mathrm{a}}$. Thus $\theta$ will increase from low values if activity is high and decrease from high values if activity is low.

The time courses of $a$ and $\theta$ for the full three-variable model resulting from the interaction of the $a-d$ fast subsystem and
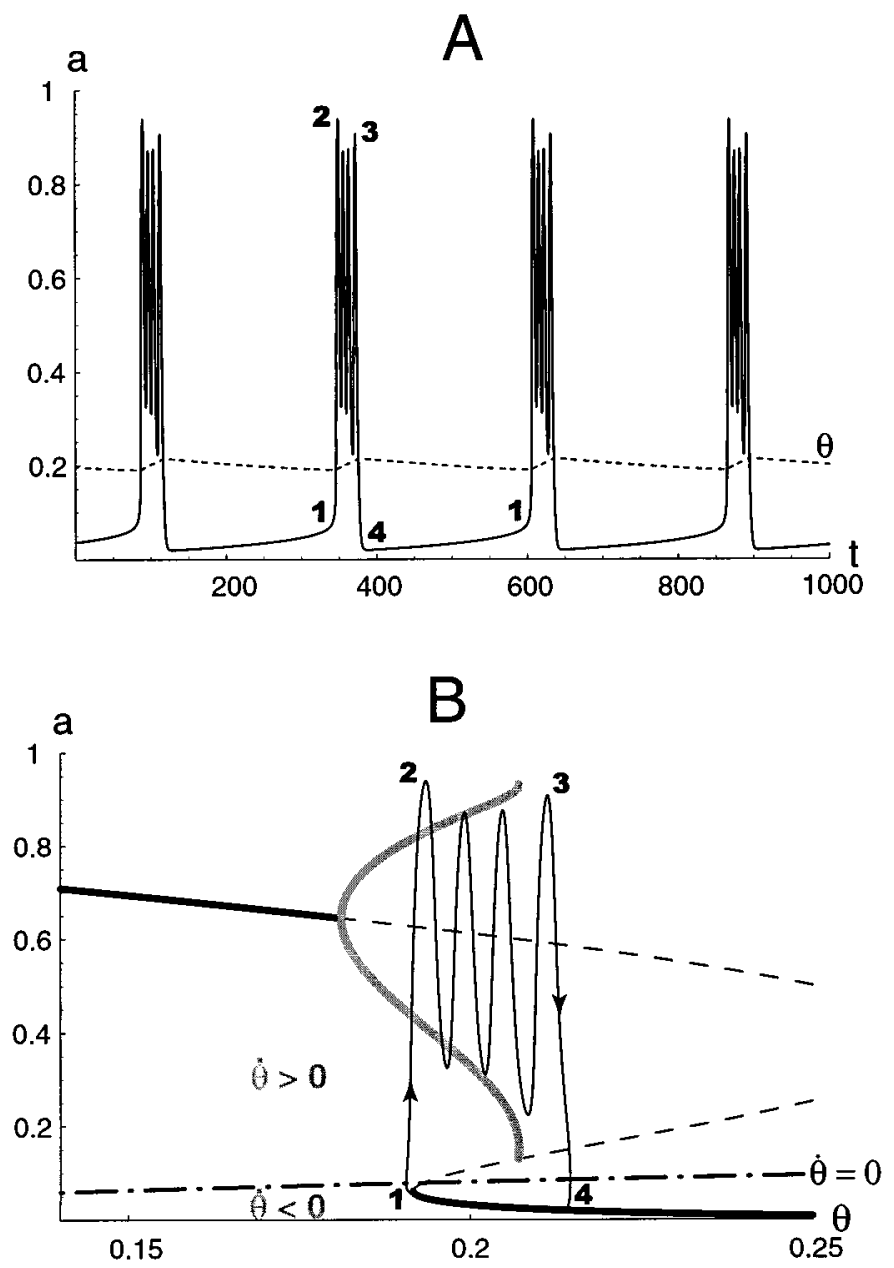

Figure 8. $A$, Time courses of $a$ and $\theta$ for the $\theta$-model. During an episode $\theta$ increases, until the episode stops. $B$, Corresponding trajectory in the $(a, \theta)$ plane, superimposed on the bifurcation diagram. Also plotted is the $\theta$-nullcline (dot-dashed curve). Below this nullcline, the trajectory flows leftward $(\dot{\theta}<0$, recovery during the silent phase); above it the trajectory flows rightward ( $\dot{\theta}>0$, depression during an episode). The points numbered $1,2,3$, and 4 correspond to the same states of the network on both $A$ and $B$. During an episode, the trajectory passes sequentially 1-2-3-4; during a silent phase it goes from 4 to 1 .

the slow $\theta$ subsystem are shown in Figure $8 A$. As expected, the behavior is episodic, with rhythmic cycling during episodes. The system's trajectory actually lives in the three-dimensional phase space, $a-d-\theta$. In Figure $8 B$ we project the trajectory onto the $(a-\theta)$ plane, enabling us to see the dynamic behavior along with the static bifurcation diagram from Figure $7 A$. We see qualitatively what we anticipated in the preceding paragraphs, with $\theta$ covering the bistable range back and forth. Although the trajectory does not exactly follow the bifurcation diagram, it would if we had made $\tau_{\theta}$ larger.

It is important to emphasize that both onset and termination of the episodes are controlled by the value of $\theta$, as shown from the trajectory in the $(a-\theta)$ plane. Also shown in Figure $8 B$ is the $\theta$-nullcline, the set of points for which $\dot{\theta}=0$, that is, $\theta=\theta_{\infty}(a)$. Below this nullcline, $\dot{\theta}<0$, therefore $\theta$ is decreasing, and conversely, for any point above the nullcline $\theta$ is increasing. Thus, $\theta$ decreases during the silent phase and increases during an episode. Obviously, the position of the $\theta$-nullcline relative to the $a-d$ bifurcation diagram is critical for the existence and timing of episodic rhythmicity (see below). 
This graphical representation allows one to easily predict/ understand the effect of parameter variations on the episodic rhythm. Suppose the cellular adaptation is recruited more easily (say, $\theta_{\theta}$ is decreased). This lowers the $\theta$-nullcline. If it drops too much it will intersect the Z-curve's lower branch. At this intersection, all variables, including $\theta$, are at a stable steady state of low activity; the network will remain silent. On the other hand, if the $\theta$-nullcline is too high, $\theta$ can stop increasing before the end of the episode, and the network might enter continuous oscillation. Therefore, the position of the nullcline, or in other words, the value of $a$ for which the average cellular excitability in the network starts to get depressed, has to be within a certain range for episodic oscillations to occur.

The episode durations and silent phase lengths depend on two factors. First, they depend on the range of values that $\theta$ covers between the beginning and end of the episodes, that is, the horizontal distance between points 4 and 1 on Figure $8 B$. Increasing this range dilates both the episode and silent phase in the same way, because the paths $1-2-3-4$ and $4-1$ are increased accordingly. As illustrated below, this range can be changed by varying any single parameter of the fast subsystem. Second, the episode durations and silent phase lengths depend on the velocity with which $\theta$ varies. Because this velocity is inversely proportional to $\tau_{\theta}$, an increase in this time constant increases similarly the time it takes to depress the network and the recovery period, so both duration and silent phase change by the same factor. However, this velocity is also proportional to the horizontal distance between the current point in the $a-\theta$ plane and the $\theta$-nullcline, because $\tau_{\theta} \dot{\theta}=\theta_{\infty}(a)-\theta$. Therefore, if the nullcline is changed [that is, the function $\theta_{\infty}(a)$ is changed], velocities are modified. However, this change in the velocity depends on the activity state (high or low) of the system. For example, if the nullcline is moved down, the velocity decreases during the silent phase because $\theta_{\infty}(a)-\theta$ is reduced, but increases during the episode because $\theta_{\infty}(a)-\theta$ is increased. Therefore, episode and silent phase lengths may be affected in opposite ways when a parameter of the slow subsystem is changed. The distinction between the effects of parameters of the fast versus slow subsystems will be important subsequently to distinguish the two models.

\section{Long-term synaptic depression: s-model}

As was considered previously, it is also possible to interpret the slow form of activity-dependent depression as arising synaptically rather than from a slow modification of cellular thresholds. Indeed, this mechanism has been proposed to account for the slow recovery of synaptic potentials after an episode (Fedirchuk et al., 1999). Calling $s$ the new, slow variable, the system becomes:

$$
\begin{aligned}
& \tau_{\mathrm{a}} \dot{a}=a_{\infty}(n \cdot s \cdot d \cdot a)-a \\
& \tau_{\mathrm{d}} \dot{d}=d_{\infty}(a)-d \\
& \tau_{\mathrm{s}} \dot{s}=s_{\infty}(a)-s .
\end{aligned}
$$

As for $d$, a high value of $s$ (near 1 ) means "not depressed," so $s_{\infty}$ is a decreasing function of $a$. Note that the fraction of nondepressed synapses is now $s \cdot d$, and the effective connectivity is $n \cdot s \cdot d$. The activity obtained with this model is qualitatively similar to the one obtained with the $\theta$-model, as shown in Figure 9. Here again, the onset and termination of episodes are controlled by the slow depression variable $(s)$, therefore silent phase and episode durations are determined in the same way. The reason the $\theta$-model and the $s$-model are so similar is that variations of $s$
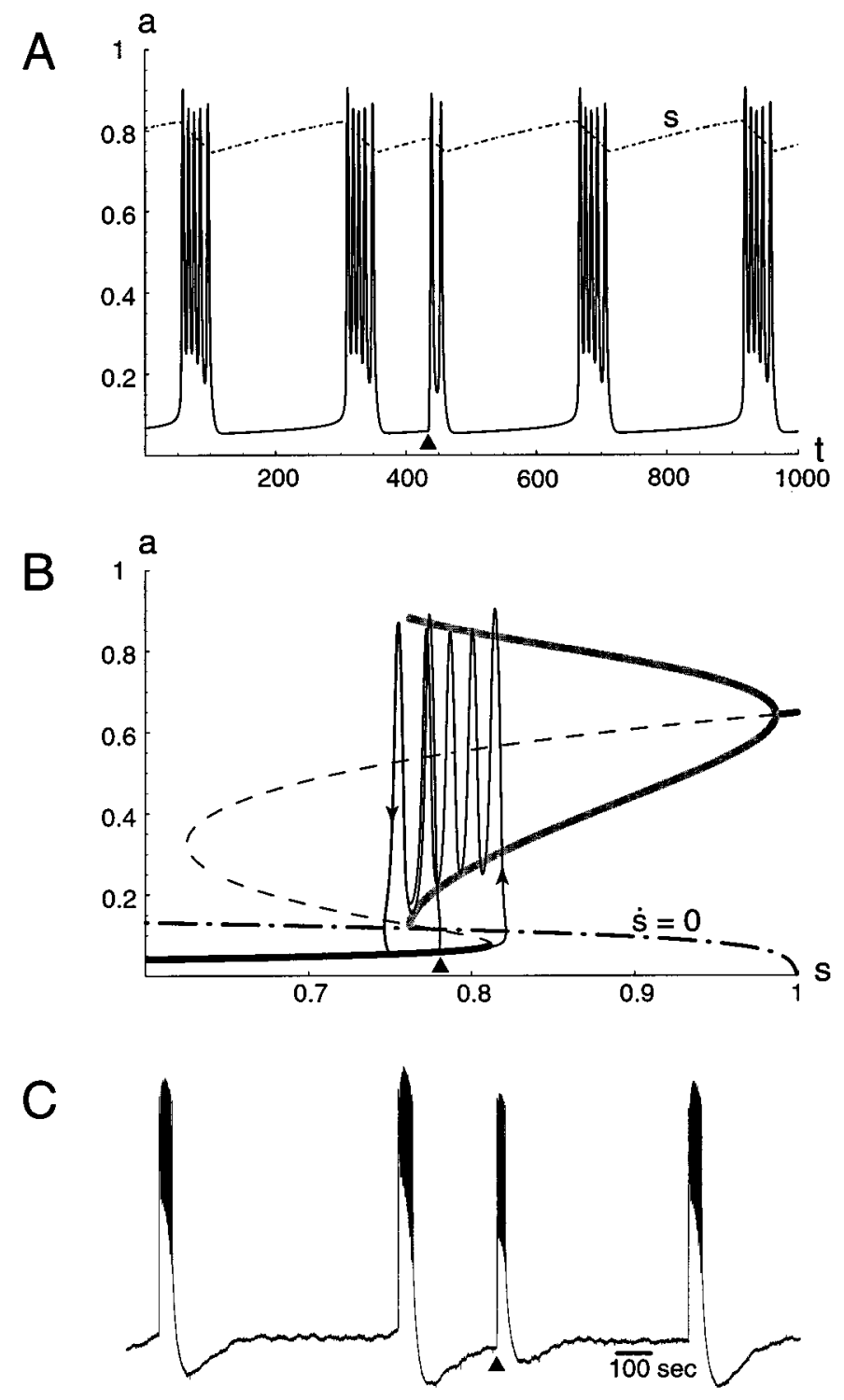

Figure 9. $A$, Time courses of $a$ and $s$ for the $s$-model. During an episode $s$ decreases, until the episode stops. As for the $\theta$-model (Fig. 7B), the cycle period increases near the end of an episode. A little after $t=400$, a stimulation was applied, which triggered an episode. This episode was shorter because recovery of $s$ was not complete. $B$, Corresponding trajectory in the $(a, s)$ plane, superimposed with the bifurcation diagram and the s-nullcline (dot-dashed curve). C, Recordings from an E10 cord. Stimulating quickly after a spontaneous episode triggers a short episode. Black triangles indicate time of stimulation.

induce the same qualitative changes in the dynamics of the fast subsystem as do variations of $\theta$. Note that because depression is associated with smaller values of $s$, in contrast to the $\theta$-model where large $\theta$ means larger depression, the bifurcation diagram of Figure $9 B$ has opposite (left/right) orientation to that of Figure $8 B$.

\section{Simulation experiments with the models}

To further characterize the models' behaviors and compare them with experimental data, a few manipulations were conducted. The model network can be perturbed by a brief stimulation. It is also important to understand how the model is affected by varying its parameters, because the parameter variations may be identified with experimental manipulations or with evolving system conditions that occur during development. In addition to the compar- 

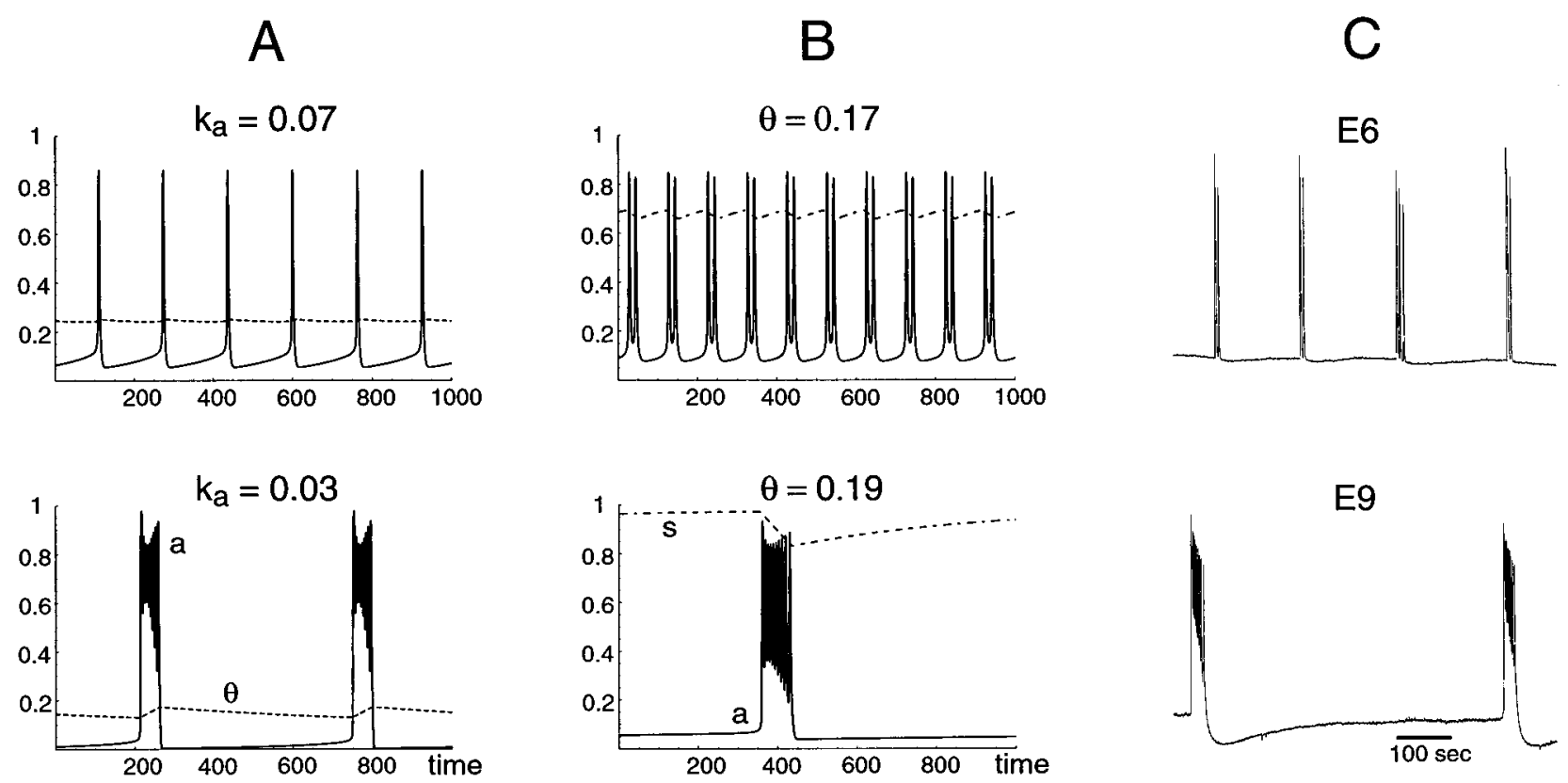

Figure 10. Time courses of activity (solid curves) and slow process (dashed curves) for different values of a parameter of the fast subsystem. $A$, Increasing the slope of $a_{\infty}$ increases both interval and episode duration ( $\theta$-model). The intermediate case $k_{\mathrm{a}}=0.05$ was shown on Figure $8 A$. Other parameter values are given in Table 1. $B$, For the $s$-model, increasing $\theta$ leads to longer silent phases and longer episodes. Notice how the increases in interval and duration are paralleled by an increase in the range covered by the slow variable. The intermediate case $\theta=0.18$ was shown on Figure $9 A$. Other parameter values are given in Table 1. $C$, Spontaneous activity recorded from chick embryonic cord at two stages of development showing the increases in silent phase and episode duration. The scale bar applies to both traces.

ison with experimental data, the following simulations offer some predictions that are testable experimentally.

Activity resetting. In the chick spinal cord, it is easy to trigger an episode during a silent phase with a brief electrical stimulus. In addition, if the stimulation is applied shortly after a spontaneous episode, the evoked episode is much shorter than the average spontaneous episode, as illustrated on Figure $9 C$. This effect was observed on all preparations tested $(>20)$. It is to be expected if the silent phase is a period of recovery from depression, and it is indeed a characteristic feature of the models presented here. We have illustrated this point for the $s$-model on Figure $9 A, B$. Again exploiting the graphical representation of Figure $9 B$, we see that to evoke an episode the stimulus must be substantial enough to bring the activity above the S-curve's middle branch. Otherwise, activity returns immediately to the low steady state. Because the vertical distance between the unstable steady state and low-activity state is highest just after an episode and progressively declines, the smaller the amount of time after an episode, the harder it is to successfully evoke a new episode, in agreement with experiments. These results are also observed with the $\theta$-model because it relies on the same mechanism of episode generation.

Changing a parameter of the fast subsystem. The main effect of changing one parameter of the fast $a-d$ subsystem is to change the values of the slow variable at the beginning and/or end of episodes (or, equivalently, at the end and beginning of silent phases) by deforming the shape of the bifurcation diagram and, in many cases, without moving it much relative to the slow variable's nullcline. Therefore, as mentioned above (section describing the $\theta$-model), silent phase and episode duration are affected in the same way, because the slow variable runs through the same range during the episode and the silent phase.

Figure 10 illustrates the effects of changing parameters in the fast subsystem: the steepness of $a_{\infty}(a)$ for the $\theta$-model (Fig. 10A) and the value of $\theta$ for the $s$-model $(B)$. Increasing the steepness (decreasing $k_{\mathrm{a}}$; see Materials and Methods) leads to larger interval and episode durations, higher cycling frequency, and larger tonic component. Making $a_{\infty}$ steeper also results in a lower level of (background) activity during the silent phase. Increasing $\theta$ in the $s$-model produces similar effects. These increases in interval and episode duration, cycling frequency, and tonic component are observed experimentally with development, as shown in Figures 1 and 10C [see also O'Donovan and Landmesser (1987)]. For instance, activity at E4-5 consists of single cycle episodes, lasting just a few seconds, but recurring with intervals of $\sim 2$ min (Milner and Landmesser, 1999). By E10-11, episodes typically last $\sim 60$ sec, with intervals of 15-20 min.

To understand more physiologically how such a change in a parameter of the fast subsystem affects both episodes and intervals in the same way, let us consider the example of varying $\theta$ for the $s$-model (Fig. 10B). To keep the argument simple, we exaggeratedly assimilate the effect on episode termination to the effects on the high-activity states. Obviously, if the cellular threshold $\theta$ is increased, $s$ will need to reach a higher value to start an episode, because neurons will require a higher level of excitation to start firing. However, the value of $s$ that terminates the episode will not be increased as much by the increase of $\theta$. The reason is that during an episode neurons receive huge synaptic inputs, bringing them far above threshold. A small change in $\theta$ therefore does not affect very much the activity during an episode. In other words, the high-activity states are rather insensitive to changes in $\theta$, as can be seen from the saturating shape of $a_{\infty}$ (Fig. 2C) for large inputs. This is also illustrated in the $a-d$ plane; note how the bottom part of the $a$-nullcline is much more shifted to the right than the top part when $\theta$ is increased (Figs. $4 B, 5 B$ ). The consequence of the need to recover more from depression before starting an episode, although the level of depression terminating 

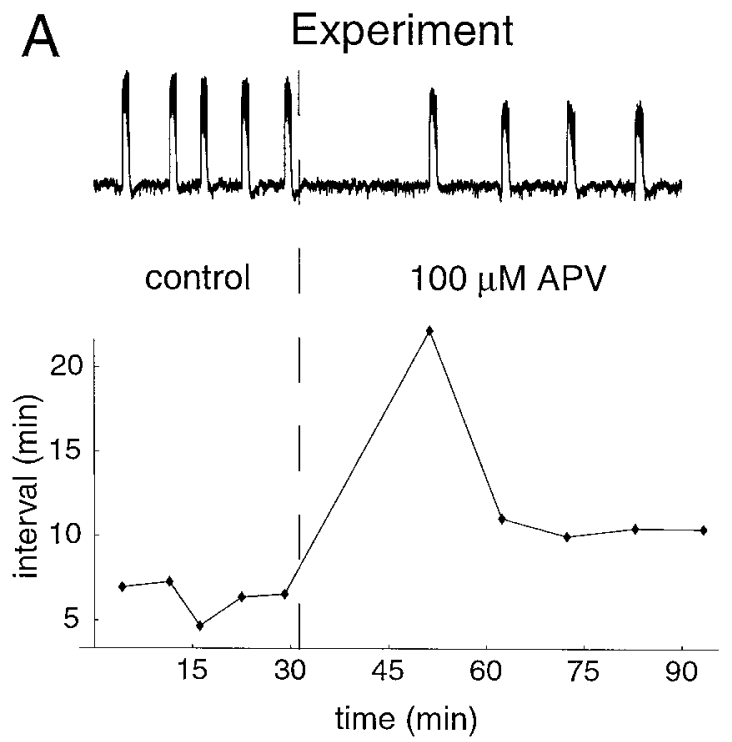
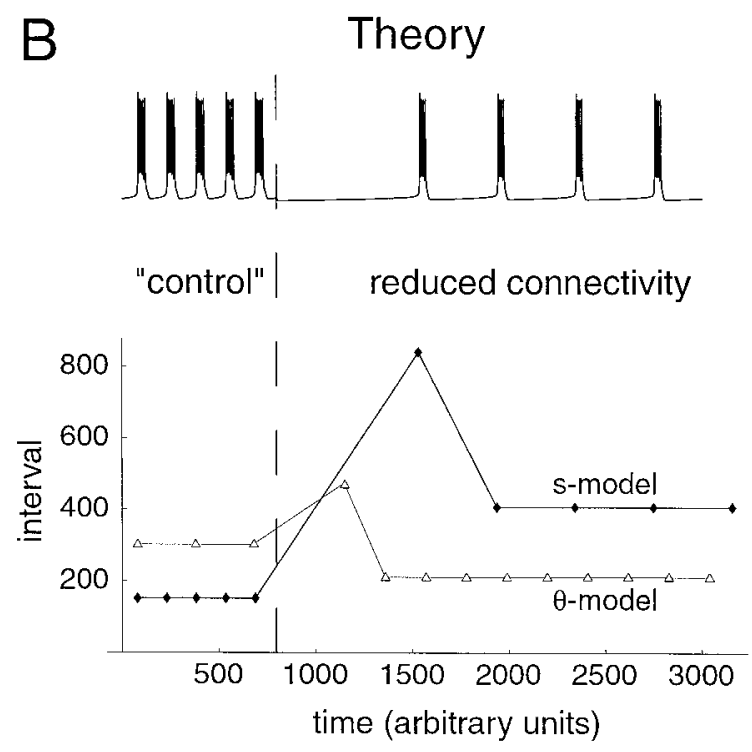

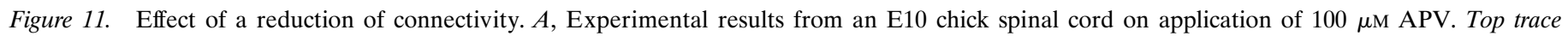

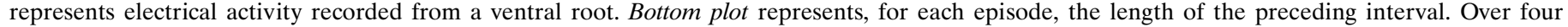

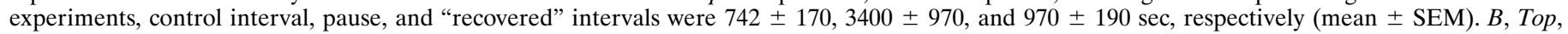

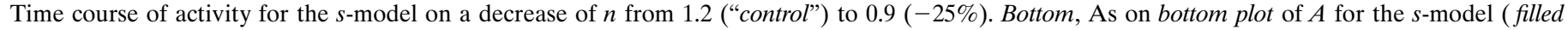
symbols) and the $\theta$-model (open triangles).

the episode is not changed much, is that intervals will be longer, and so will episodes be. The increased level of effective connectivity needed to start an episode for increased cellular threshold also means increased synaptic drive at the beginning of an episode and thus explains the larger tonic components for higher $\theta$. A lower level of "background" activity just after an episode is also attributable to the increased value of $\theta$, because for a higher cellular threshold, in the absence of network activity the number of cells firing randomly will be even smaller. Again this can be seen from the $a_{\infty}$ curve (Fig. $2 C$ ), which shifts to the right when $\theta$ increases, or in the $a-d$ plane by comparing Figures $4 B$ and $5 B$. Note how the lower branch of the $a$-nullcline, and therefore the low state, is almost down to 0 for the higher $\theta$.

We have also seen that a decrease in $\tau_{\mathrm{d}}$ leads to similar effects, and so does an increase in $\theta_{\mathrm{d}}$ (data not shown). These results suggest that developmental changes that affect the fast subsystem (time scale $<1 \mathrm{sec}$ ) could be responsible for the increase of episode duration and interepisode interval (time scale minutes), as well as an increase of the tonic component, with age. This prediction is testable by comparing the time constant of fast depression (for example) at different stages of development. The model also suggests that these developmental changes are accompanied by a reduction in the background activity during the silent phase. Again this could be tested experimentally with intracellular or optical recordings.

\section{Distinguishing the two models}

To summarize our previous results, we have constructed two models of episodic oscillatory activity. They share common rhythmogenic properties. Their cycle-generating fast subsystems, reflecting the interplay of a regenerative activity attributable to recurrent excitatory connections and (fast) synaptic depression, are bistable (coexistent states of cyclic high activity and near quiescence). The episodic nature of the activity results from the addition of a slow, activity-dependent process, in one case a slow modulation of cellular properties $(\theta$-model $)$ and in the other case a slow synaptic depression ( $s$-model). Both models have similar dynamics, where the slow process switches the network between low and high activity states. As a result, changing a parameter of the fast subsystem affects interepisode interval and episode duration in the same way for both models.

How can we distinguish between the two models? What experiment would manipulate a parameter that has different effects on the $\theta$-model than on the $s$-model? We have seen that varying a parameter of the slow subsystem could affect interval and duration in opposite ways. Therefore, varying a parameter that is in the fast subsystem for one model and in the slow subsystem for the other model could have different effects on the models. In this last section, we show that the connectivity parameter, $n$, affects both models in a different way and also has experimental relevance. Applying the graphical analysis used in the previous sections, we show that only the $s$-model can explain adequately the experimental data, specifically the effects induced by a reduction of synaptic transmission.

Because $n$ can be identified with the number of connections in the network, it is suitable for experimental manipulation. One way to accomplish this is to antagonize synaptic receptors. For instance, it has been shown previously that on application of the NMDA receptor antagonist APV (alone or in conjunction with CNQX) the activity stops for a period of time but then recovers, with moderately longer interepisode intervals than in control conditions (accompanied by a slight decrease in episode duration) (Barry and O’Donovan, 1987; Chub and O'Donovan, 1998a). We therefore have redone these experiments (using only APV) and compared the results with a decrease of $n$ for both models (Fig. 11). All of four experiments on E9-10 preparations showed the transient cessation of activity after drug application, followed by recovered activity with moderately longer intervals than control (after recovery, there was also a slow decrease of the intervals with time, such that in one of the experiments the average interval after recovery was equal to the average control interval).

Both models reproduce the transient cessation of activity after a decrease in connectivity, although it is less marked for the 

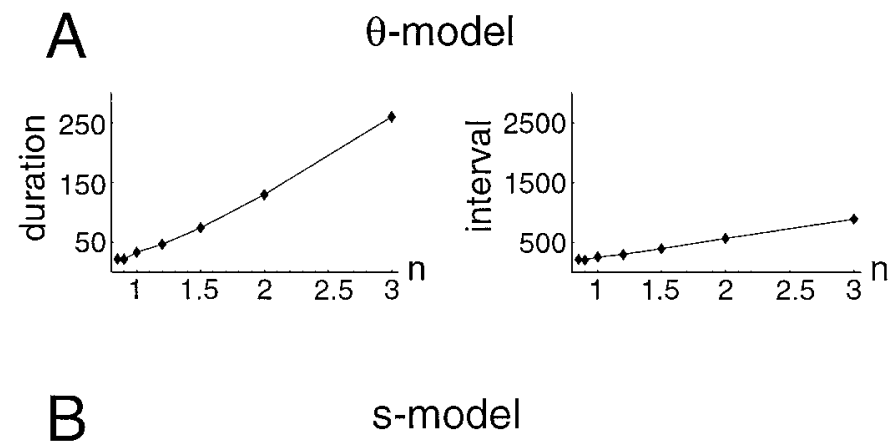

s-model
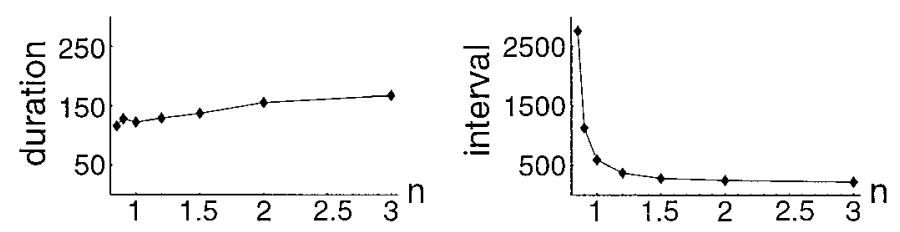

Figure 12. Episode duration and interepisode interval as a function of connectivity for the two models. $A, \theta$-model. Both duration and interval increase with increased $n$, with a relatively smaller effect on interval. $B$, $s$-model. Although duration increases slightly, interval decreases with increasing $n$.

$\theta$-model. More importantly, the following intervals are paradoxically shorter than control for the $\theta$-model, whereas they are larger for the $s$-model, as observed experimentally. Also, episode duration decreases markedly for the $\theta$-model, in contrast to the slight decrease observed experimentally. These results suggest that the $s$-model is in better agreement with the data than the $\theta$-model. The effects of varying $n$ on both interepisode interval and episode duration are summarized in Figure 12. The models clearly differ in the way intervals are affected. For the $\theta$-model, episode duration and interepisode interval are both decreased when connectivity is reduced, whereas for the $s$-model, interval increases for a moderate range of $n$ and duration only slightly decreases.

The fast/slow dissection approach and associated bifurcation diagrams enable us to understand these results. Figure 13 shows explicitly how changing the connectivity, $n$, affects the solution skeleton of the $a-d$ fast subsystem for the $\theta$-model. With greater connectivity, activity can start for a higher value of $\theta$ and can also be sustained for higher values of $\theta$, because of the higher synaptic drive. However, the value of $\theta_{c}$ (at an episode's end) increases more than the value of $\theta$ at the Z-curve's left knee (at an episode's beginning). This means that the bistable range, which is covered by the slow variable $\theta$ during episode and silent phase, is increased for increased $n$, as shown on the Figure (horizontal bars). Therefore, because the same range is covered during the episode and during the silent phase, both silent phase and episode duration are increased. Note however that the range covered by $\theta$ is also slightly shifted to the right for larger $n$. This slightly increases the horizontal distance from the $\theta$ nullcline (and therefore speed) during a silent phase and decreases it during an episode. Therefore this further increases the duration of an episode but limits the extent of an interval, as shown on Figure 12. There is a lower limit on the connectivity that allows episodic rhythmicity. If $n$ is too small the Z-curve's lower branch intersects the $\theta$-nullcline, forming a steady state of the whole system. Therefore the network remains at this low activity level. If the $\theta$-nullcline were higher, $n$ could be decreased further, but for $n$ too low the bistability disappears and so does episodic activity.

We can now explain in a more intuitive way the effect of
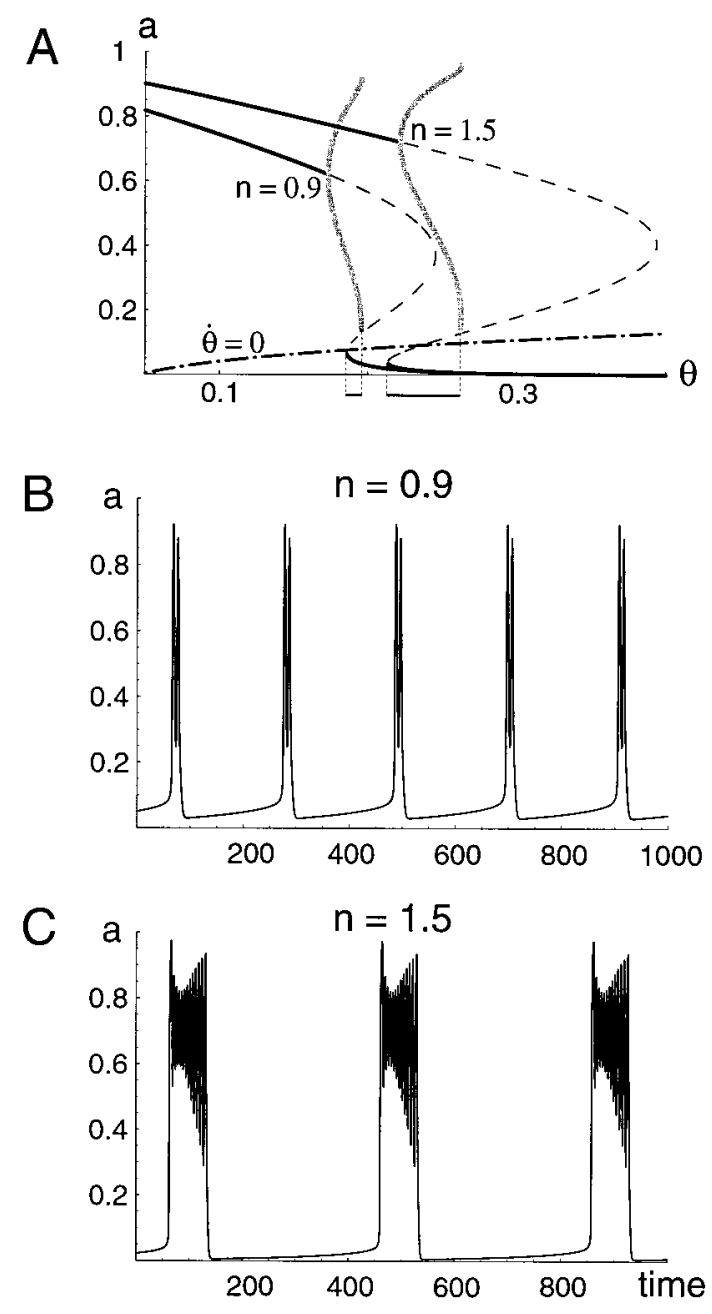

Figure 13. Effects of changing connectivity in $\theta$-model. $A$, Bifurcation diagrams corresponding to two different values of $n$. Changing $n$ mainly scales the diagram and slightly moves it to the right. The main effect is on the range of bistability, indicated by horizontal bars. Therefore silent phase and duration are changed in the same direction. $B$, Time course of network activity for $n=0.9$. $C$, Time course for $n=1.5$.

connectivity changes. Increasing connectivity implies a higher synaptic drive, and therefore episodes are sustained until the cellular threshold reaches a higher critical value than for lower $n$. However, the value of $\theta$ that has to be reached to start an episode does not increase as much because during the silent phase activity is low, and therefore increasing connectivity does not increase synaptic drive as much as during an episode. Because with higher connectivity episodes start at approximately the same value of $\theta$, but end for a higher value $\theta_{c}$, their duration is increased. In turn, silent phases start at a higher value of the threshold, so they increase in duration too. Note how an increase of connectivity for the $\theta$-model is analogous to an increase in $\theta$ for the $s$-model (Fig. $10 B)$. The duality of effects of $\theta$ and $n$ on the two models is discussed in the Appendix. It is this duality that allows us to distinguish the two models.

In the $s$-model, $n$ can be identified as a parameter of the slow subsystem. Changing this parameter can thus affect episode duration and interepisode interval in opposite ways. To demonstrate this, let us define a new variable $\tilde{s}=n \cdot s$. The system then becomes: 

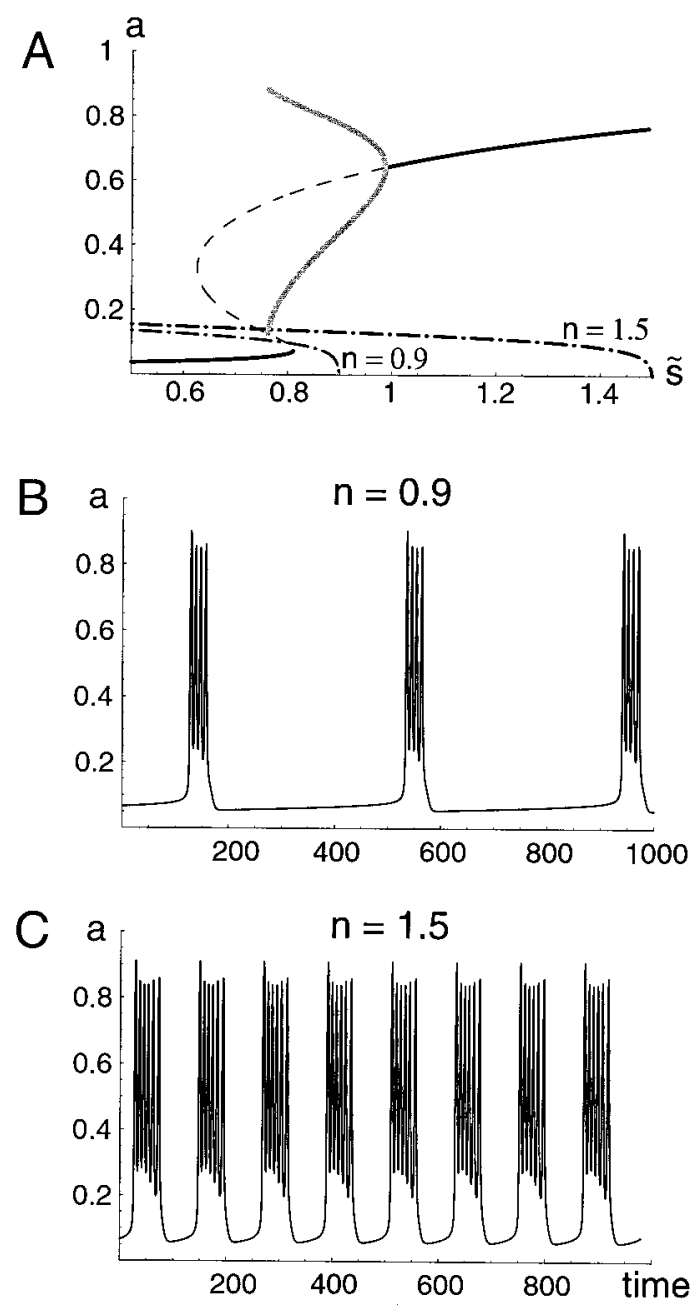

Figure 14. Effects of changing connectivity in $\tilde{s}$ version of $s$-model. $A$, Bifurcation diagram with $\tilde{s}$-nullclines corresponding to two different values of $n$. Changing $n$ only changes the maximal value of $\tilde{s}$ covered by the nullcline. If $n$ is further decreased below 0.85 , the $\tilde{s}$-nullcline intersects the low steady-state branch so episodic activity ceases. $B$, Time course of network activity for $n=0.9$. $C$, Time course for $n=1.5$.

$$
\begin{aligned}
\tau_{\mathrm{a}} \dot{a} & =a_{\infty}(\tilde{s} \cdot d \cdot a)-a \\
\tau_{\mathrm{d}} \dot{d} & =d_{\infty}(a)-d \\
\tau_{\mathrm{s}} \dot{\tilde{s}} & =n \cdot s_{\infty}(a)-\tilde{s},
\end{aligned}
$$

so that a variation of $n$ results only in a multiplicative scaling of the $\tilde{s}$-nullcline, as shown in Figure $14 A$. From this diagram it is easily seen that the range of $\tilde{s}$ does not change with $n$, but the relative speeds of $\tilde{s}$ are affected. During the silent phase the distance between the trajectory and the $\tilde{s}$-nullcline is smaller for smaller $n$, implying a lower velocity during recovery. Therefore, when connectivity is lowered, interval increases. At the same time, episode duration slightly decreases (Figs. 12, 14B,C). The reason for only a small effect on duration is that during activity, $s_{\infty}(a) \simeq 0$, so multiplying it by $n$ does not change it much, implying that the part of the $\tilde{s}$ nullcline relevant to high activity is moved only slightly leftward (for decreased $n$ ) compared with the part corresponding to the recovery phase.

After application of the glutamate antagonists there is a very large interval, immediately followed by regular occurrence of episodes. It has been hypothesized previously that the lack of activity for a long period of time $(\sim 1 \mathrm{hr})$ could lead to an upregulation of the GABA/glycinergic synapses (Chub and O'Donovan, 1998a). The strengthening of these synapses would compensate for the blockade of the glutamatergic synapses. Our graphical approach helps us to interpret this result with the $s$-model in a different way. As shown in Figure 15, when $n$ is decreased, the whole diagram is shifted instantaneously to the right, that is, to larger values of $s$. However, just after the manipulation the variable $s$, because it is slow, will be unchanged. The system's state drops to the low-activity branch of the shifted $\mathrm{S}$-curve (the only attractor at this $s$-value). Before an episode can be initiated, $s$ has to recover to a much larger value (the shifted right knee), hence the long period of inactivity. Once $s$ is in the right range, episodes occur regularly as in control conditions. Therefore, the model suggests that the hypothesized upregulation of GABA/glycinergic synapses may simply be a shift to a lower level of depression.

Finally, there is a corollary to this transient large interval after a decrease in connectivity. If $n$ is brought back to its original value, in other words, if the drugs are washed out, $s$ has to decrease back to its original range. Assuming that the washout is immediate, $s$ has to decrease while activity is in the high state. Therefore the model predicts that after washout there should be a very long episode. To test this prediction we have examined the length of the episodes after washing out APV (CNQX was not applied because it washes out too slowly). We did not find such a dramatic increase in the next episode's duration, probably because the drug does not wash out instantaneously. In two cases, there was a longer episode, but the general effect was a transient reduction of the silent phase. This can be interpreted with the model as a gradual change from the S-curve structure on the right of Figure 15 to that on the left. As the diagram drifts leftward and the trajectory during a silent phase is moving to the right, they intersect, and episodes are started more often than usual, until $s$ reaches the control range. If we measure the percentage of active phase (the duration divided by the length of the preceding interval), the model predicts a transient decrease in this percentage after drug application and a transient increase after washout. This is what is observed experimentally in all four preparations (data not shown).

\section{DISCUSSION}

We have analyzed models that describe the activity of a randomly connected, excitatory network with activity-dependent network depression. The models reproduce many features of the spontaneous activity generated by developing spinal networks and make a number of experimentally testable predictions. We have used graphical methods to provide insights into the behavior of the models and show that they can account for the newly discovered short-term plasticity (Chub and O’Donovan, 1998a) exhibited by developing spinal networks.

In the first part of Discussion, we will consider the models and the insights that they have provided into the genesis and properties of spontaneously active spinal networks. In addition, we will identify processes of the models with their putative biological equivalents, and we will then discuss some of the specific predictions made by using the models. In the second part of Discussion, we will explain some limitations of the models and those aspects of the network behavior that the models cannot account for in their present form. Finally, we will discuss extensions of the model to other spontaneously active networks and compare it to 
Figure 15. Simulation with $s$-model of applying and then washing out an excitatory synaptic antagonist. The bifurcation diagram for two values of $n$ and the corresponding trajectory in the $(a, s)$ plane are plotted. Assume connectivity is initially "high" $(n=1.5)$. When connectivity is decreased (after drug application), the bifurcation diagram switches to the one on right. Therefore the trajectory falls to the lowactivity steady state and migrates along the lower branch (for $n=0.9$ ). Because it must travel far rightward, to the shifted lower knee, there is a very long "interval" before the next episode can occur. Once the new range of $s$ allowing for activity has been reached, episodes occur regularly. If connectivity is suddenly increased back to control (washout), the bifurcation diagram switches back to the left. Thus, the trajectory migrates leftward, but this time following the high activity state, hence a very long "episode" before the control range of $s$ values is reached.

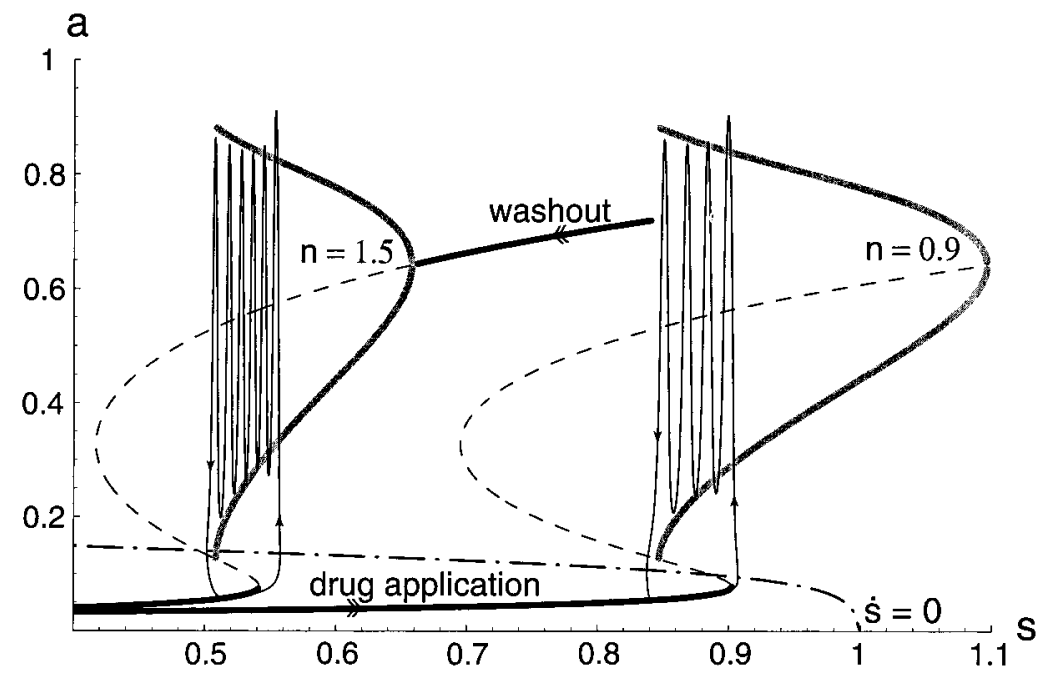

other models that have been developed to account for spontaneous network activity.

\section{Model predictions and insights into the genesis of spontaneous activity by spinal networks}

We consider first the slow form of network depression that controls the onset and termination of episodes because it is in this area that most of the experimental data have been accumulated. What is the physiological correlate of this slow depression? As mentioned earlier, a variation of both neuronal threshold and synaptic strength are compatible with the experimental findings. Intracellular recordings have revealed a slow depolarization between episodes [N. Chub and M. O'Donovan, unpublished result; see O'Donovan (1999)], and evoked synaptic potentials are depressed after an episode and progressively recover in the interepisode interval (Fedirchuk et al., 1999). The $s$-model favors synaptic depression as the primary mechanism because it duplicates the experimental findings when glutamate antagonists are applied to the network. Indeed the $s$-model provides an important insight into the mechanisms of the recovery of activity in the presence of excitatory blockers because it suggests that it is a consequence of the presence of slow synaptic depression rather than requiring the involvement of an additional mechanism. In the model, the mechanism for rhythmicity within an episode is a fast form of synaptic depression. Its time constant is of the order of $\tau_{\mathrm{a}}$, but longer, so that the feedback inhibition provided by $d$ is delayed, which allows for the cycling observed during episodes. At present, we have only preliminary evidence to support the presence of fast depression in the synapses responsible for the rhythmic drive to motoneurons and interneurons (P. Wenner, unpublished observations). However, previous studies have indicated the existence of a fast depression of the monosynaptic connection between muscle afferents and motoneurons (Lee and O'Donovan, 1991). The time constant for this depression in E18 embryos was 200-300 msec, which is in the appropriate range for oscillatory behavior. However, the time constant for synaptic depression is very dependent on developmental maturity, and further studies will be necessary to establish whether the time constant of the active synapses is in the range predicted by the model.

\section{Limitations of the present model}

Our modeling framework uses two classes of slow activitydependent network depression ( $s$ or $\theta$ ), which deterministically control the onset and termination of episodes. As a consequence, for a given set of parameter values the episode and interval lengths will be constant (except for small regimes of parameter values where chaotic behavior might occur). However, this contradicts our experimental observations showing that these durations vary from episode to episode. Some form of synaptic noise could affect the onset of episodes, because intracellular recording from ventrally located spinal neurons has shown the presence of action potentialindependent synaptic noise that peaks in amplitude just before the episode (Chub and O'Donovan, 1998b). Experimentally, we have equated the slow depression to the reduction in the amplitude of synaptic potentials that follows an episode. The maximal depression of these evoked potentials occurs $\sim 1$ min after an episode. On the falling phase of the episode depolarization, the evoked potentials can actually be facilitated (Fedirchuk et al., 1999). This suggests that an additional process might be involved in episode termination. Finally, we cannot exclude the possibility that both synaptic depression and some form of activity-dependent cellular modulation affect network excitability.

Another area in which the models fail to predict the measured network behavior is in the response to the bath-applied $\mathrm{GABA}_{\mathrm{A}}$ and glycine antagonists bicuculline and strychnine. These drugs cause a marked decrease in episode duration, whereas interepisode intervals become less regular (but not necessarily longer). This is not expected from a decrease of $n$ in the $s$-model. This discrepancy between the modeling and the experimental results may arise because the actions of these drugs cannot simply be viewed as simply a reduction in connectivity ( $n$ parameter in the model). Indeed, although GABA and glycine depolarize spinal neurons and are thought to be functionally excitatory, activation of $\mathrm{GABA}_{\mathrm{A}}$ receptors can produce a shunt that prevents neuronal firing (O’Donovan, 1989). Blockade of these receptors could therefore increase neuronal firing frequency during an episode, depressing synapses faster than in control conditions. Moreover, there is evidence that extracellular GABA produces a persistent conductance in spinal neurons, so that bath-applied bicuculline may lead to an increase in input resistance (Chub and O'Donovan, unpublished results) and thus to an increased sensitivity to synaptic noise.

\section{Comparison with other models of spontaneous activity}

There are several other models of spontaneous activity that also rely on some form of slow activity-dependent depression. For example, Staley et al. (1998) have proposed that transmitter depletion terminates epileptiform discharges induced by inhibitory 
blockade or elevated extracellular $\mathrm{K}^{+}$in hippocampal slices. They suggest that during an episode, the number of available transmitter vesicles $(\mathrm{Nr})$ decreases until it reaches a threshold value $T h r$ where activity can no longer be sustained. During the following silent phase, they propose that synapses recover exponentially and that a new episode starts when $\mathrm{Nr}$ reaches a given value that depends on network excitability and $T h r$. This scheme is similar to the $s$-model. In the model of Staley et al. (1998), an increase in network excitability decreases the duration of both the episode and the interepisode, as in the $s$-model when $\theta$ is decreased. Also, it can be shown that $N r / T h r$ is similar to our variable $s$, and therefore that an increase of $T h r$ is similar to a decrease of connectivity in our model: interepisode interval is increased, but episode duration is not affected substantially. It is worth noting that in the scheme of Staley et al. (1998), the time constant of transmitter depletion during a burst is much faster than the time constant of recovery during the silent phase. This allows the interburst interval to vary independently of burst duration. In our model there is only one time constant $\tau_{\mathrm{s}}$ for both depression and recovery, so scaling it affects both interval and duration in the same way. For this reason, in our model it is difficult to achieve the experimentally observed interval/duration ratios $(>10)$. It is possible to get high ratio values for the $s$-model for low values of connectivity. We do not claim, however, that this is an additional piece of evidence in favor of the $s$-model as presented here, because we could also achieve higher ratios by allowing $\tau_{\mathrm{s}}$ (or $\tau_{\theta}$ in the $\theta$-model, for that matter) to be activity dependent, being greater during the silent phase.

Feller et al. (1996) have used a cellular refractory period to account for the limited domains of spontaneous retinal waves. Still unknown are the precise mechanisms of this refractoriness and whether synaptic depression plays a role in limiting wave frequency or extent. We have suggested a way to distinguish between these two possibilities by manipulating connectivity (or neuronal threshold, cf. Appendix), but it is not clear how it could be applied to the retinal network.

In conclusion, we have shown that a highly idealized model with very few assumptions is capable of generating spontaneous activity such as the one observed in the experimental preparation. Despite and because of the simplifications, the model allows a basic understanding of the processes involved in generation of episodic, rhythmic activity. Whether the slow activity-dependent process is a modulation of cellular excitability or a depression of intercellular connections does not change the qualitative behavior of the model. However, the two models show different responses to a change in connectivity or in cellular excitability. This can be used experimentally to help decide which is the better model to apply to a given preparation.

\section{APPENDIX}

\section{Condition for oscillations of the fast subsystem}

We consider the fast subsystem and rewrite it as follows:

$$
\begin{aligned}
& \dot{a}=A(a, d) \\
& \dot{d}=D(a, d),
\end{aligned}
$$

where $A(a, d)=a_{\infty}(n \cdot d \cdot a)-a$ and $D(a, d)=\left(d_{\infty}(a)-d\right) / \tau$ with $\tau=\tau_{\mathrm{d}} / \tau_{\mathrm{a}}$. We will derive the condition associated with the destabilization of a steady state and birth of cyclic oscillations (via Hopf bifurcation) around the high-activity steady state. Two aspects of this condition are that the steady state must be on the $a$-nullcline's middle branch and that the negative feedback from depression must be sufficiently slow.
The stability of a steady state $(\bar{a}, \bar{d})$ is analyzed in the traditional way (Rinzel and Ermentrout, 1998) using linear perturbation theory. Consider the effect of a small perturbation $(x, y)$ from the steady state and determine whether it grows or decays. That is, we write $a=\bar{a}+x$ and $d=\bar{d}+y$. We assume the perturbations to be small enough so that

$$
A(a, d) \approx A(\bar{a}, \bar{d})+x \cdot \frac{\partial A}{\partial a}(\bar{a}, \bar{d})+y \cdot \frac{\partial A}{\partial d}(\bar{a}, \bar{d})
$$

(and the same approximation applies to $D$ ).

The system becomes:

$$
\begin{aligned}
& \dot{x}=x \cdot \frac{\partial A}{\partial a}(\bar{a}, \bar{d})+y \cdot \frac{\partial A}{\partial d}(\bar{a}, \bar{d}) \\
& \dot{y}=x \cdot \frac{\partial D}{\partial a}(\bar{a}, \bar{d})+y \cdot \frac{\partial D}{\partial d}(\bar{a}, \bar{d}) .
\end{aligned}
$$

This is a system of two linear differential equation with constant coefficients. Therefore the solution is a linear combination of two exponentials, $\exp \left(\lambda_{1} t\right)$ and $\exp \left(\lambda_{2} t\right)$. As long as the real part of the $\lambda_{\mathrm{i}}$ values are negative, the perturbation will decay to 0 ; the steady state is stable in this case. The steady state loses stability in an oscillatory manner when a control parameter is varied so that the $\lambda_{\mathrm{i}}$ values as a complex pair cross the imaginary axis, from $\operatorname{Re}(\lambda)<0$ to $\operatorname{Re}(\lambda)>0$. At the critical point, $\lambda_{1}+\lambda_{2}=$ 0 (each has zero real part), and this happens when:

$$
\frac{\partial A}{\partial a}(\bar{a}, \bar{d})+\frac{\partial D}{\partial d}(\bar{a}, \bar{d})=0 .
$$

Recalling the definition of $A$ and $D$, we have that at the critical point of destabilization:

$$
n \bar{d} a_{\infty}^{\prime}(n \bar{d} \bar{a})-1=1 / \tau .
$$

This is the necessary and sufficient condition (along with a few technical details) for a Hopf bifurcation of periodic solutions to occur (Rinzel and Ermentrout, 1998). Thus, the steady state will be an unstable spiral only if:

$$
n \bar{d} a_{\infty}^{\prime}(n \bar{d} \bar{a})-1>1 / \tau .
$$

We glean several interpretations from this condition as follows. First, we note that the quantity on the left side is equal to $-n \bar{a} a_{\infty}^{\prime}(n \bar{d} \bar{a}) M$, where $M$ is the slope of the $a$-nullcline. Because this term has to be greater than $1 / \tau, M$ must be negative, i.e., the steady state must be on the middle branch of the $a$-nullcline (as in Figs. $4 B, C$, and $6 A, B)$. This is a geometric statement, but to guarantee instability we need the parametric aspect as well. The cells' activation function must be steep enough, and their thresholds should not be too spread out. That is, we must have high-gain positive feedback. Also, if $n$ or $d$ is too small, destabilization will not occur. Importantly, the depression time constant has to be large enough. If the negative feedback is too fast, then the steady state can be stable even though it is on the middle branch. If the gain of the positive feedback is increased, the time constant of the negative feedback can be decreased and still not preclude destabilization.

The above condition does not guarantee that the fast subsystem can oscillate, only that there is a high-activity, unstable steady state. In the example of Figure 6, increasing $\tau$ destabilizes the high state so that oscillations can occur. However, further increasing $\tau$ increases the oscillation amplitude and can cause the periodic to hit the intermediate steady state and then disappear. Therefore, in that case there is a range of values for $\tau$ that allow oscillations (1.4-2.4). In the 


\begin{tabular}{|c|c|c|}
\hline$\theta$-model & $s$-model & Effect \\
\hline $\begin{array}{l}\text { Decreased } \\
\text { connectivity }\end{array}$ & $\begin{array}{l}\text { Increased } \\
\text { tonic excitation }\end{array}$ & $\begin{array}{l}\text { Shorter interval, } \\
\text { shorter episode }\end{array}$ \\
\hline $\begin{array}{l}\text { Decreased } \\
\text { tonic excitation }\end{array}$ & $\begin{array}{l}\text { Decreased } \\
\text { connectivity }\end{array}$ & $\begin{array}{l}\text { Longer interval, } \\
\text { shorter episode }\end{array}$ \\
\hline
\end{tabular}

The first line corresponds to changes that affect interval and duration in the same way; the second line indicates changes that affect interval and duration in opposite ways.

example of Figure 4, however, there is no lower steady state, so for any value of $\tau$ larger than 6 there will be oscillations.

\section{Dual effects of connectivity and threshold}

We have noted previously, in "Distinguishing the two models," how a change in connectivity in the $\theta$-model is analogous to a change of $\theta$ in the $s$-model. In the latter, an increase in $\theta$ leads to a higher value of $s$ before an episode is started, which increases the silent phase and in turn increases episode duration. Similarly, an increase in connectivity in the $\theta$-model allows the episodes to continue for a larger value of $\theta$, increasing episode duration and in turn increasing the silent phase. Each of these parameters is identified as belonging to the fast subsystem of the respective model.

We have also shown that a decrease of connectivity induces an increase in interval for the $s$-model, whereas it decreases the interval in the $\theta$-model. This allows us to distinguish between the two models. Here, we show that manipulating the average cellular threshold, or bias excitation to the neurons (that is, depolarization from a source external to the network), allows a similar distinction.

Changing $\theta$ in the $s$-model is equivalent to changing the bias excitation. For the $\theta$-model, we represent the influence of an external excitation by introducing a parameter, $\theta_{0}$, adding it to the argument $n \cdot d \cdot a$ of $a_{\infty}$. As we saw for $n$ in the $s$-model, we see here that the bias excitation $\theta_{0}$ can be viewed as a parameter of the slow subsystem in the $\theta$-model, by defining the new variable $\tilde{\theta}=\theta-\theta_{0}$. The effect of $\theta_{0}$ is to shift the $\theta$-nullcline by $-\theta_{0}$, which for $\theta_{0}$ positive decreases the silent phase (slightly) while it increases the duration. Table 2 summarizes the types of transformations that are analogous in the $s$ - and $\theta$-models. We believe that this might help one to decide whether the episodic activity observed in other preparations is dominated by cellular or synaptic properties.

It is interesting to note that if the activation function $a_{\infty}$ were a step function, as used in some models, it would not be possible to distinguish between the $\theta$-model and the $s$-model, because a change in both connectivity and threshold would have the same effect on the activation function.

The behavior of the $\theta$-model is similar to that of a cell-based network model of a respiratory rhythm generator developed by Butera et al. (1999). In the respiratory model, cell-cell coupling is also purely excitatory and a fraction of cells are endogeneous (bursting) oscillators. For strong enough coupling, this model behaves as the $\theta$-model: increasing the tonic excitation to the network shortens interval but increases duration, whereas decreasing connectivity decreases both interval and duration.

\section{REFERENCES}

Barry M, O'Donovan M (1987) The effects of excitatory amino acids and their antagonists on the generation of motor activity in the isolated chick spinal cord. Dev Brain Res 36:271-276.

Butera R, Rinzel J, Smith J (1999) Models of respiratory rhythm generation in the pre-bötzinger complex: II. populations of coupled pacemaker neurons. J Neurophysiol 82:398-415.
Butts D, Feller M, Shatz C, Rokhsar D (1999) Retinal waves are governed by collective network properties. J Neurosci 19:3580-3593.

Chance F, Nelson S, Abbott L (1998) Synaptic depression and the temporal response characteristics of V1 cells. J Neurosci 18:4785-4799.

Cherubini E, Gaiarsa J-L, Ben-Ari Y (1991) GABA: an excitatory transmitter in early postnatal life. Trends Neurosci 14:515-519.

Chub N, O'Donovan M (1998a) Blockade and recovery of spontaneous rhythmic activity after application of neurotransmitter antagonists to spinal networks of the chick embryo. J Neurosci 18:294-306.

Chub N, O'Donovan M (1998b) Miniature postsynaptic currents and their modulation by spontaneous activity in the E11-E12 chick embryo spinal cord. Soc Neurosci Abstr 2:1789.

Fedirchuk B, Wenner P, Whelan P, Ho S, Tabak J, O'Donovan M (1999) Spontaneous network activity transiently depresses synaptic transmission in the embryonic chick spinal cord. J Neurosci 19:2102-2112.

Feller M, Wellis D, Stellwagen D, Werblin F, Shatz C (1996) Requirement for cholinergic synaptic transmission in the propagation of spontaneous retinal waves. Science 272:1182-1187.

Feller M, Butts D, Aaron H, Rokhsar D, Shatz C (1997) Dynamic processes shape spatiotemporal properties of retinal waves. Neuron 19:293-306.

Ho S, O’Donovan M (1993) Regionalization and intersegmental coordination of rhythm-generating networks in the spinal networks of the chick embryo. J Neurosci 13:1354-1371.

Lee M, Koebba M, O’Donovan M (1988) The development of sensorimotor synaptic connections in the lumbosacral cord of the chick embryo. J Neurosci 8:2530-2543.

Milner L, Landmesser L (1999) Cholinergic and GABAergic inputs drive patterned spontaneous motoneuron activity before target contact. J Neurosci 19:3007-3022.

O'Donovan M (1989) Motor activity in the isolated spinal cord of the chick embryo: synaptic drive and firing pattern of single motoneurons. J Neurosci 9:943-958.

O'Donovan M (1999) The origin of spontaneous activity in developing networks of the vertebrate nervous system. Curr Opin Neurobiol 9:94-104.

O’Donovan M, Chub N (1997) Population behavior and self-organization in the genesis of spontaneous rhythmic activity by developing spinal networks. Semin Cell Dev Biol 8:21-28.

O'Donovan M, Landmesser L (1987) The development of hindlimb motor activity studied in the isolated spinal cord of the chick embryo. J Neurosci 7:3256-3264.

O’Donovan M, Wenner P, Chub N, Tabak J, Rinzel J (1998) Mechanisms of spontaneous activity in the developing spinal cord and their relevance to locomotion. Ann NY Acad Sci 860:130-141.

Pinto D, Brumberg J, Simons D, Ermentrout G (1996) A quantitative population model of whisker barrels: re-examining the Wilson-Cowan equations. J Comput Neurosci 3:247-264.

Rinzel J, Ermentrout G (1998) Analysis of neural excitability and oscillations. In: Methods in neuronal modeling. Cambridge, MA: MIT.

Rinzel J, O'Donovan M (1997) A model of rhythm generation based on synaptic depression. Abstract for the Computational Neuroscience Meeting, Big Sky, Montana, July.

Ritter A, Wenner P, Ho S, Whelan P, O’Donovan M (1999) Activity patterns and synaptic organization of ventrally located interneurons in the embryonic chick spinal cord. J Neurosci 19:3457-3471.

Senn W, Wyler K, Streit J, Larkum M, Lüscher H, Mey H, Müller L, Stainhauser D, Vogt K, Wannier T (1996) Dynamics of a random neural network with synaptic depression. Neural Net 9:575-588.

Sernagor E, Chub N, Ritter A, O'Donovan M (1995) Pharmacological characterization of the rhythmic synaptic drive onto lumbosacral motoneurons in the chick embryo spinal cord. J Neurosci 15:7452-7464.

Staley K, Longacher M, Bains J, Yee A (1998) Presynaptic modulation of CA3 network activity. Nat Neurosci 1:201-209.

Streit J (1993) Regular oscillations of synaptic activity in spinal networks in vitro. J Neurophysiol 70:871-878.

Strogatz S (1994) Nonlinear dynamics and chaos. Redding, MA: Addison Wesley.

Tabak J, Senn W, O’Donovan M, Rinzel J (1999) Comparison of two models for pattern generation based on synaptic depression. Neurocomputing 26-27:551-556.

Traub R, Jefferys J, Whittington M (1994) Enhanced NMDA conductance can account for epileptiform activity induced by low $\mathrm{Mg}^{2+}$ in the rat hippocampal slice. J Physiol (Lond) 478:379-393.

Wilson H, Cowan J (1972) Excitatory and inhibitory interactions in localized populations of model neurons. Biophys J 12:1-24. 